\title{
Prevalence of Depression in Coronary Artery Bypass Surgery: A Systematic Review and Meta-Analysis
}

\author{
María Correa-Rodríguez ${ }^{1,2}\left(\mathbb{D}\right.$, Moath Abu Ejheisheh ${ }^{1}\left(\mathbb{D}\right.$, Nora Suleiman-Martos ${ }^{3, *(\mathbb{D}}$, \\ María José Membrive-Jiménez ${ }^{4}\left(\mathbb{D}\right.$, Almudena Velando-Soriano ${ }^{5}{ }^{(\mathbb{D}}$, \\ Jacqueline Schmidt-RioValle ${ }^{1,+}+$ and José Luis Gómez-Urquiza ${ }^{1,+}+$ (i) \\ 1 Faculty of Health Sciences, University of Granada, Avenida de la Ilustración N. 60, 18016 Granada, Spain; \\ macoro@ugr.es (M.C.-R.); moad.ibrahem@hotmail.com (M.A.E.); jschmidt@ugr.es (J.S.-R.); \\ jlgurquiza@ugr.es (J.L.G.-U.) \\ 2 Instituto de Investigación Biosanitaria, IBS, 18012 Granada, Spain \\ 3 Faculty of Health Sciences, University of Granada, Campus Universitario de Ceuta, \\ C/Cortadura del Valle s/n, 51001 Ceuta, Spain \\ 4 University Hospital of Ceuta. Institute of Health Management. C/Colmenar s/n, 51003 Ceuta, Spain; \\ mariajose.membrive@gmail.com \\ 5 University Hospital Virgen de las Nieves. Andalusian Health Service. Av. de las Fuerzas Armadas 2, \\ 18014 Granada, Spain; srtavelando@correo.ugr.es \\ * Correspondence: norasm@ugr.es \\ + These authors contributed equally to this work.
}

Received: 4 February 2020; Accepted: 25 March 2020; Published: 26 March 2020

\begin{abstract}
Coronary artery bypass graft surgery (CABG) might adversely affect the health status of the patients, producing cognitive deterioration, with depression being the most common symptom. The aim of this study is to analyse the prevalence of depression in patients before and after coronary artery bypass surgery. A systematic review and meta-analysis was carried out, involving a study of the past 10 years of the following databases: CINAHL, LILACS, MEDLINE, PsycINFO, SciELO, Scopus, and Web of Science. The total sample comprised $n=16,501$ patients. The total number of items was $n=65$, with $n=29$ included in the meta-analysis. Based on the different measurement tools used, the prevalence of depression pre-CABG ranges from $19-37 \%$, and post-CABG from $15-33 \%$. There is a considerable presence of depression in this type of patient, but this varies according to the measurement tool used and the quality of the study. Systematically detecting depression prior to cardiac surgery could identify patients at potential risk.
\end{abstract}

Keywords: coronary artery bypass graft; depression; mental health; meta-analysis; prevalence; surgery; systematic review

\section{Introduction}

Coronary artery disease (CAD) is one of the leading causes of death in developed countries, and it is associated with deteriorated quality of life, disability, and premature death [1]. The usual surgical treatment involves coronary artery bypass graft surgery (CABG). This technique is based on revascularisation by diverting blood flow to other arteries to increase the blood supply to the heart muscle [2].

Although CABG surgery increases life expectancy [3], it is associated with multiple physical complications, including myocardial infarction, stroke, and even kidney failure [4]; in addition to psychological consequences, such as mood disorders, fatigue, weakness, stress, anxiety, and depression [5]. 
Short-term recovery factors include a longer hospital stay, pain, and infection, which may predispose towards cognitive disorders, like anxiety and depression [6]. In the long term, it is estimated that at least $25 \%$ of patients will experience deteriorated quality of life after a CABG; and, it even doubles the post-surgery risk of future cardiac events and mortality related to high levels of anxiety and depression [7].

In particular, depression is considered to be one of the main reasons for reduced well-being, having a negative impact on a patient's quality of life, as well as their social and family life. It is a strong risk factor for mortality, being related to the occurrence of new cardiac events and reduced functionality up to six months post-CABG surgery, increasing the risk of hospital readmission in up to $20 \%$ of patients, due to complications including infection, arrhythmia, and volume overload [8].

Diagnosis is sometimes difficult, since symptoms, such as loss of appetite, sleep cycle disturbance, and constant fatigue, may be superimposed over the same symptoms that were derived from surgery. For this reason, determining the degree to which a CABG can affect a patient's mental, psychological, and social skills, and, specifically, analysing the level of depression, requires the use of multiple tools validated during a clinical interview $[9,10]$.

A number of factors seem to influence the relationship between depression and CABG, including biological alterations (cardiac rhythm alterations, tone of cardiac muscle, hormone levels, and reduced brain perfusion) [11]. However, in many cases, the high prevalence of mood disorders cannot be explained by the severity of the illness, but is instead related to psychosocial factors, such as socioeconomic status, lifestyle (adherence to the recommended diet or prescribed treatment), or the level of social support [12].

Even though the effects of CABG have been studied in terms of morbidity, mortality, and organ function, the effect or influence it has on mood disorders, like depression, remains unclear. It seems that depression predicts how much a patient's health will deteriorate. Therefore, it is extremely important to assess how the disease affects a patient, as this can influence the therapeutic benefit and, consequently, which interventions and care are prioritised, and which self-care strategies are implemented both before and after surgery $[13,14]$.

Although depression is considered to have a negative impact on patient recovery, few studies have examined the association between CABG and depression. Some systematic reviews have analysed the risk factors [15], and there are also reports regarding the effect of certain interventions [16,17]. However, to our knowledge, no meta-analysis studies that include a prevalence analysis have been exclusively undertaken on CABG patients.

Describing the levels of depression in CABG patients is essential for analysing the importance of this surgery with regard to depression levels. The purpose of this work is, therefore: (1) to analyse the prevalence of depression in patients both before and after CABG surgery; and, (2) to analyse the depression levels over time.

\section{Materials and Methods}

The data were extracted and analysed based on the recommendations of preferred reporting items for systematic review and meta-analysis (PRISMA) 2015 [18].

\subsection{Search Strategy}

A search was conducted of CINAHL, LILACS, MEDLINE, PsychINFO, SciELO, Scopus, and Web of Science in January 2020. MeSH descriptors were used, with the search strategy being: “(depression OR depressive disorder) AND (coronary artery bypass grafting)".

\subsection{Inclusion and Exclusion Criteria}

The inclusion criteria were the following: (1) full text of quantitative primary studies; (2) men and women aged over 18; (3) no psychiatric pathology or illness; (4) CABG surgery; (5) study of depression 
levels prior to or after CABG; (6) the use of a validated scale; (7) written in English, Portuguese, Spanish, or French; and, (8) published in the last 10 years.

The exclusion criteria were the following: (1) paediatric population; (2) a different type of cardiac surgery that was not exclusively CABG (CABG with valve replacement); (3) measurement of depression in relatives; (4) patients with an active treatment deriving from a psychiatric disorder; (5) data from duplicate articles in previous studies; and, (6) no depression data extracted using a validated scale.

\subsection{Selection of Articles and Information Analysis}

Firstly, two authors checked the title and abstract, and, secondly, the full text of the article. A third author was consulted in the case of discrepancy.

For the meta-analysis, we selected the data from those studies that used the same measurement tool, since the inclusion of several measurement tools would not permit the results to be integrated, due to different scores.

\subsection{Data Extraction}

The following variables were recorded: (1) data on the study (author, year, country); (2) type of CABG (first time, elective or emergency); (3) study characteristics (sample, type of study, sex, and follow-up time); (4) measurement tool; and, (5) mean, standard deviation, prevalence of depression. For clinical trials or quasi-experimental studies, we selected only the levels of depression prior to the programme intervention (baseline) or those relating to the control group.

We used the intraclass correlation coefficient to analyse coding reliability, obtaining an average value of 0.97 (minimum $=0.93$; maximum $=1$ ), and the Cohen's kappa coefficient with a mean value of 0.94 (minimum $=0.92$; maximum $=1$ ).

\subsection{Assessment of Quality and Measurement of Bias}

Two independently authors assessed the quality of the studies, consulting with a third party in the event of a disagreement.

For observational studies (cohort and cross-sectional), we followed the guidelines in "Strengthening the Reporting of Observational Studies in Epidemiology" (STROBE) [19]. We followed the standards in the Cochrane Collaboration Risk of Bias tool for clinical trials [20].

We used a second quality assessment tool to analyse the level of evidence in accordance with the recommendations of the Oxford Centre for Evidence-Based Medicine [21] (Table 1).

\subsection{Data Synthesis and Statistical Analysis}

The meta-analysis included those studies that used the same tool for measuring depression. We performed six meta-analyses using a random-effects model and two meta-analyses using a fixed-effect model, for prevalence levels and confidence intervals, through the statistical package StatsDirect (version 3, StatsDirect Ltd., Cambridge, UK).

We used $\mathrm{I}^{2}$ to analyse the heterogeneity, grouping values into low (25\%), moderate (50\%), or high (75\%) heterogeneity [22]. The publication bias was assessed using Egger's test.

\section{Results}

The search yielded a total of $n=1874$ articles. After reading the title and abstract, 662 were excluded. Figure 1 shows the study selection process. 


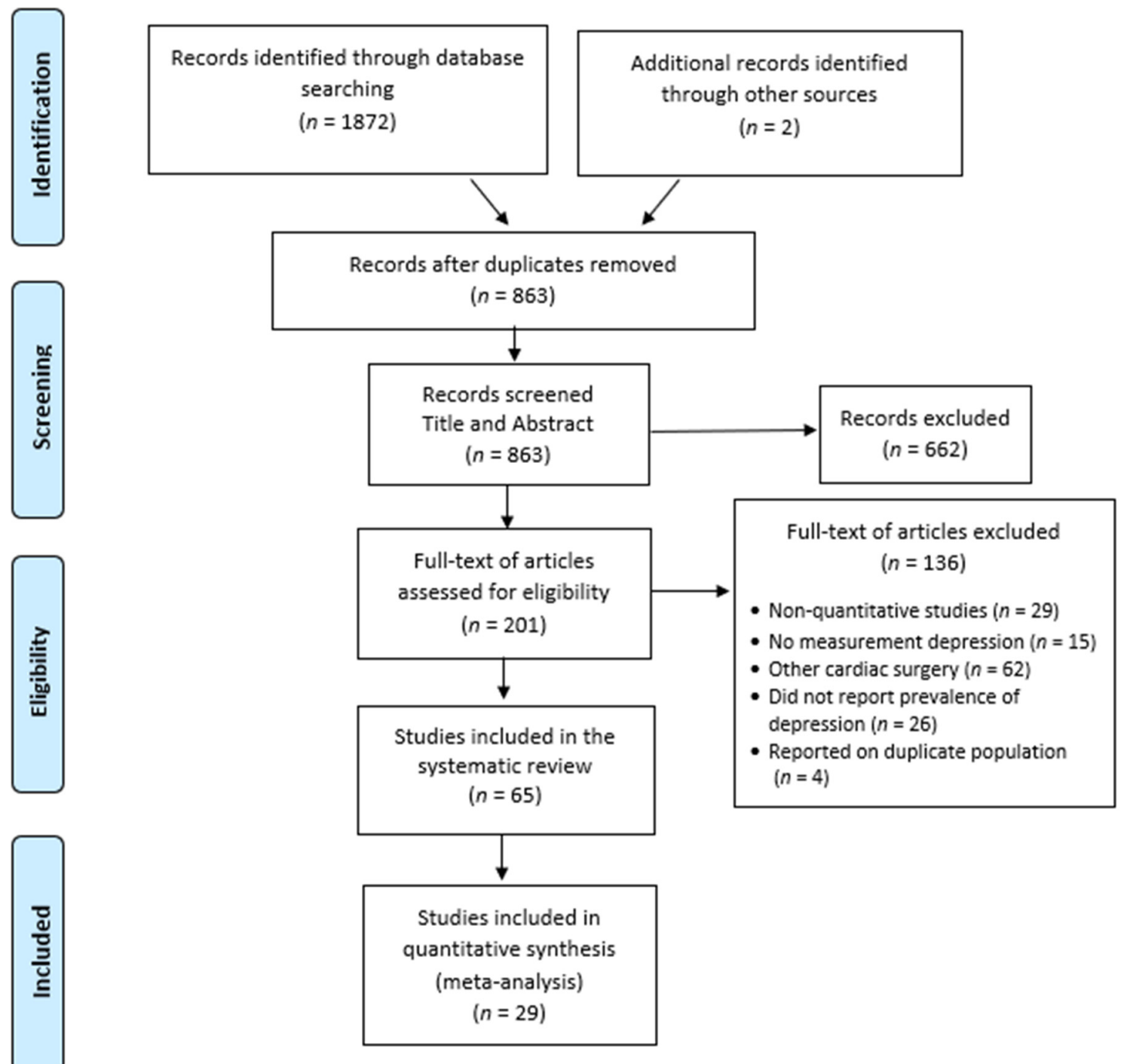

Figure 1. Preferred reporting items for systematic review and meta-analysis (PRISMA) flow-chart of included studies.

\subsection{Characteristics of Included Studies}

The total sample comprised 16,501 patients, predominantly male $(n=54)$. Most of the studies were cohort studies $(n=34)$, followed by cross-sectional studies $(n=12)$. Thirteen studies evaluated the levels prior to surgery, 23 after surgery, and 29 both before and after. Most of the studies were carried out in the USA $(n=17)$, followed by Germany $(n=7)$, Iran $(n=7)$, and Australia $(n=6)$ (Table 1). The depression follow-up ranged from a month prior to surgery (since the pre-assessment clinic appointment) [23] up to six years after surgery [24,25]. 
Table 1. Studies reporting prevalence and levels of coronary artery bypass graft surgery (CABG) depression.

\begin{tabular}{|c|c|c|c|c|c|c|c|}
\hline \multirow{2}{*}{$\begin{array}{c}\text { Study } \\
\text { CABG (First, Elective, Emergency) }\end{array}$} & \multirow{2}{*}{$\begin{array}{l}\text { Design and } \\
\text { Sample }\end{array}$} & \multirow{2}{*}{$\begin{array}{c}\text { Depression } \\
\text { Screening } \\
\text { Instrument }\end{array}$} & \multirow{2}{*}{$\begin{array}{c}\text { Timing of } \\
\text { Assessment }\end{array}$} & \multicolumn{2}{|c|}{ M (SD)/Prevalence } & \multirow{2}{*}{ Main Results } & \multirow{2}{*}{$\mathrm{EL} / \mathrm{RG}$} \\
\hline & & & & Pre & Post & & \\
\hline $\begin{array}{l}\text { Abbott et al., [26] } \\
\text { USA } \\
\text { Elective CABG }\end{array}$ & $\begin{array}{c}\text { RCT } \\
n=226 \\
83 \% \text { male }\end{array}$ & HADS & After CABG & - & $\begin{array}{l}\text { Cluster 1: } 5.9(4.3) \\
\text { Cluster 2: } 8.2(4.8) \\
\text { Cluster 3: } 11.8(6.9)\end{array}$ & $\begin{array}{l}\text { Elderly patients with more } \\
\text { symptoms and chronic } \\
\text { illnesses are more prone to } \\
\text { depression }\end{array}$ & $1 \mathrm{a} / \mathrm{A}$ \\
\hline $\begin{array}{c}\text { Aburuz, [27] } \\
\text { Jordan } \\
\text { Elective CABG }\end{array}$ & $\begin{array}{c}\text { Cohort } \\
n=227 \\
78 \% \text { male }\end{array}$ & HADS & $\begin{array}{l}\text { 2-weeks before, } \\
\text { 1-month after }\end{array}$ & $\begin{array}{c}12.76(6.80) \\
\text { Normal: } 57.26 \% \\
\text { Mild: } 11.90 \% \\
\text { Moderate-severe: } \\
30.84 \%\end{array}$ & $\begin{array}{c}11.11(6.78) \\
\text { Normal: } 59.47 \% \\
\text { Mild: } 13.66 \% \\
\text { Moderate-severe: } 26.87 \%\end{array}$ & $\begin{array}{l}\text { Pre-operative depressive } \\
\text { symptoms increased } \\
\text { postoperative hospital } \\
\text { length of stay }\end{array}$ & $2 \mathrm{c} / \mathrm{B}$ \\
\hline $\begin{array}{c}\text { Açıel, [28] } \\
\text { Turkey } \\
\text { Elective CABG }\end{array}$ & $\begin{array}{c}\text { Cohort } \\
n=65 \\
76.9 \% \text { male }\end{array}$ & BDI & $\begin{array}{c}\text { 1-day before } \\
\text { 3-7 days, } 1-\text {-month } \\
\text { after }\end{array}$ & $\begin{array}{c}\text { 8.12 (5.44) } \\
\text { Normal: } 61.5 \% \\
\text { Mild: } 30.8 \% \\
\text { Moderate: } 7.7 \% \\
\text { Severe: } 0 \%\end{array}$ & $\begin{array}{c}\text { 3rd day: } 12.43(6.36) \\
\text { Normal: } 35.4 \% \\
\text { Mild: } 40.0 \% \\
\text { Moderate: } 23.1 \% \\
\text { Severe: } 1.5 \% \\
\text { 7th day: } 11.66(6.95) \\
\text { Normal: } 40.0 \% \\
\text { Mild: } 30.8 \% \\
\text { Moderate: } 27.7 \% \\
\text { Severe: } 1.5 \% \\
\text { 1 month: } 12.29(9.08) \\
\text { Normal: } 47.7 \% \\
\text { Mild: } 26.2 \% \\
\text { Moderate: } 23.1 \% \\
\text { Severe: } 3.1 \%\end{array}$ & $\begin{array}{c}\text { Depression levels increase } \\
\text { during postoperative CABG } \\
\text { period }\end{array}$ & $2 \mathrm{c} / \mathrm{B}$ \\
\hline
\end{tabular}


Table 1. Cont

\begin{tabular}{|c|c|c|c|c|c|c|c|}
\hline \multirow{2}{*}{$\begin{array}{c}\text { Study } \\
\text { CABG (First, Elective, Emergency) }\end{array}$} & \multirow{2}{*}{$\begin{array}{l}\text { Design and } \\
\text { Sample }\end{array}$} & \multirow{2}{*}{$\begin{array}{l}\text { Depression } \\
\text { Screening } \\
\text { Instrument }\end{array}$} & \multirow{2}{*}{$\begin{array}{l}\text { Timing of } \\
\text { Assessment }\end{array}$} & \multicolumn{2}{|c|}{ M (SD)/Prevalence } & \multirow{2}{*}{ Main Results } & \multirow{2}{*}{$\mathrm{EL} / \mathrm{RG}$} \\
\hline & & & & Pre & Post & & \\
\hline $\begin{array}{l}\text { Afridi et al., [29] } \\
\quad \text { Pakistan } \\
\text { First-time CABG }\end{array}$ & $\begin{array}{c}\text { Cohort } \\
n=134 \\
84.3 \% \text { male }\end{array}$ & HAM-D & $\begin{array}{l}2 \text { days before, at } \\
\text { discharge, } \\
\text { 6-months } \\
\text { follow-up }\end{array}$ & $\begin{array}{c}98.5 \% \\
\text { Mild: } 71.6 \% \\
\text { Moderate: } 23.9 \% \\
\text { Severe: } 1.5 \% \\
\text { Very severe: } 1.5 \%\end{array}$ & $\begin{array}{c}\text { At discharge: } 80.6 \% \\
\text { Mild: } 73.9 \% \\
\text { Moderate: } 2.23 \% \\
\text { Severe: } 2.23 \% \\
\text { Very severe: } 2.23 \% \\
\text { 6-months: } 16.4 \%\end{array}$ & $\begin{array}{l}\text { Depression is commonly } \\
\text { reported before and after } \\
\text { CABG and influences the } \\
\text { quality of life of the patients }\end{array}$ & $2 \mathrm{c} / \mathrm{B}$ \\
\hline $\begin{array}{l}\text { Ajtahed et al., [30] } \\
\text { Iran } \\
\text { First-time CABG }\end{array}$ & $\begin{array}{c}\text { RCT } \\
n=75 \\
67 \% \text { male }\end{array}$ & DASS & After CABG & - & $\begin{array}{c}\text { Control group: } \\
\text { Group 1: } \\
\text { Normal: } 60 \% \\
\text { Mild: } 40 \% \\
\text { Moderate: } 24 \% \\
\text { Severe: } 12 \% \\
\text { Extremely severe: } 0 \% \\
\text { Group 2: } \\
\text { Normal: } 52 \% \\
\text { Mild: } 8 \% \\
\text { Moderate: } 32 \% \\
\text { Severe: } 0 \% \\
\text { Extremely severe: } 8 \% \\
\text { Group 3: } \\
\text { Normal: } 45 \% \\
\text { Mild: } 9.1 \% \\
\text { Moderate: } 13.6 \% \\
\text { Severe: } 13.6 \% \\
\text { Extremely severe: } 18.2 \%\end{array}$ & $\begin{array}{l}\text { Training cognitive } \\
\text { rehabilitation can improve } \\
\text { cognitive functions and } \\
\text { quality of life in patients } \\
\text { after CABG surgery }\end{array}$ & $1 \mathrm{a} / \mathrm{A}$ \\
\hline $\begin{array}{l}\text { Ammouri et al., [31] } \\
\text { Jordan } \\
\text { First-time CABG }\end{array}$ & $\begin{array}{c}\text { Cross-sectional } \\
n=100 \\
80 \% \text { male }\end{array}$ & CSS & $\begin{array}{l}\text { 2-weeks after } \\
\text { discharge }\end{array}$ & - & $3 \%$ & $\begin{array}{l}\text { Pain, leg swelling, poor } \\
\text { appetite and trouble } \\
\text { sleeping are the most } \\
\text { frequent symptoms after } \\
\text { CABG }\end{array}$ & $2 \mathrm{c} / \mathrm{B}$ \\
\hline $\begin{array}{c}\text { Amouzeshi et al., [32] } \\
\text { Iran } \\
\text { Elective CABG }\end{array}$ & $\begin{array}{c}\text { Cross-sectional } \\
n=54 \\
68 \% \text { male }\end{array}$ & BDI & $\begin{array}{l}1 \text { day before and } \\
\text { after ICU }\end{array}$ & $\begin{array}{c}11.7(7) \\
\text { Minimal: } 55.4 \% \\
\text { Mild: } 28.6 \% \\
\text { Moderate: } 16.1 \% \\
\text { Severe: } 0 \%\end{array}$ & $\begin{array}{c}\text { Male: } 31.5(10.60) \\
\text { Female: } 29.3(10.55) \\
\text { Minimal: } 0 \% \\
\text { Mild: } 17.9 \% \\
\text { Moderate: } 32.1 \% \\
\text { Severe: } 46.4 \%\end{array}$ & $\begin{array}{l}\text { No relationship between } \\
\text { age, sex, marital status, and } \\
\text { education level with } \\
\text { post-operative depression }\end{array}$ & $2 \mathrm{c} / \mathrm{B}$ \\
\hline
\end{tabular}


Table 1. Cont.

\begin{tabular}{|c|c|c|c|c|c|c|c|}
\hline \multirow{2}{*}{$\begin{array}{c}\text { Study } \\
\text { CABG (First, Elective, Emergency) }\end{array}$} & \multirow{2}{*}{$\begin{array}{l}\text { Design and } \\
\text { Sample }\end{array}$} & \multirow{2}{*}{$\begin{array}{l}\text { Depression } \\
\text { Screening } \\
\text { Instrument }\end{array}$} & \multirow{2}{*}{$\begin{array}{l}\text { Timing of } \\
\text { Assessment }\end{array}$} & \multicolumn{2}{|c|}{ M (SD)/Prevalence } & \multirow{2}{*}{ Main Results } & \multirow{2}{*}{$\mathrm{EL} / \mathrm{RG}$} \\
\hline & & & & Pre & Post & & \\
\hline $\begin{array}{c}\text { Azzopardi \& Lee, [33] } \\
\text { Australia } \\
\text { Elective CABG }\end{array}$ & $\begin{array}{c}\text { Cohort } \\
n=48 \\
85.4 \% \text { male }\end{array}$ & BDI & $\begin{array}{l}\text { Before, 6-weeks } \\
\text { after, 1-2 years } \\
\text { follow-up }\end{array}$ & $7.31(4.1)$ & 2 years: $7.90(7.1)$ & $\begin{array}{l}\text { Depression levels } 2 \text { years } \\
\text { after CABG were not severe }\end{array}$ & $2 b / B$ \\
\hline $\begin{array}{l}\text { Bay et al., [34] } \\
\text { USA } \\
\text { Elective CABG }\end{array}$ & $\begin{array}{c}\text { RCT } \\
n=170 \\
75 \% \text { male }\end{array}$ & HADS & $\begin{array}{l}\text { Baseline,1-6 } \\
\text { months after }\end{array}$ & $\begin{array}{l}\text { Control group: } \\
7.3(3.7)\end{array}$ & $\begin{array}{c}\text { Control group: } \\
\text { 1-month: } 3.0(3.1) \\
\text { 6-months: } 3.0(3.1)\end{array}$ & $\begin{array}{c}\text { A coping religious } \\
\text { intervention can reduce } \\
\text { depression levels up to } 6 \\
\text { months after surgery }\end{array}$ & $1 \mathrm{a} / \mathrm{A}$ \\
\hline $\begin{array}{l}\text { Beresnevaite et al., [35] } \\
\text { Lithuania } \\
\text { Elective CABG }\end{array}$ & $\begin{array}{c}\text { Cross-sectional } \\
n=109 \\
100 \% \text { male }\end{array}$ & SCL-90R & 1-day before after & $\begin{array}{c}63.13(8.22) \\
\text { High level: } 23 \%\end{array}$ & - & $\begin{array}{l}\text { Preoperative depression } \\
\text { score is related with a } \\
\text { length stay hospital }(\mathrm{p}< \\
0.001) \text { and perioperative } \\
\text { complications }(p<0.05)\end{array}$ & $2 b / B$ \\
\hline $\begin{array}{c}\text { Cebeci \& Çelik, [36] } \\
\text { Turkey } \\
\text { First-time CABG }\end{array}$ & $\begin{array}{c}\text { Quasi-experimental } \\
n=52 \\
80.8 \% \text { male }\end{array}$ & HADS & $\begin{array}{l}\text { 1-day before, } \\
\text { 1-day,1-week, } \\
\text { 1-month after }\end{array}$ & $8.3(3.6)$ & $\begin{array}{c}\text { At discharge: } 7.9(4.2) \\
\text { 1-week: } 8.2(4.5) \\
\text { 1-month: } 7.7(4.3)\end{array}$ & $\begin{array}{c}\text { At the time of admission, } \\
\text { patients had a higher level } \\
\text { of depression than at the } \\
\text { time of discharge }\end{array}$ & $1 \mathrm{~b} / \mathrm{A}$ \\
\hline $\begin{array}{l}\text { Chocron et al., [37] } \\
\text { France } \\
\text { First-time CABG }\end{array}$ & $\begin{array}{c}\text { RCT } \\
n=361\end{array}$ & BDI & Before CABG & $39 \%$ & - & $\begin{array}{l}\text { Antidepressant treatment } \\
\text { did not affect the morbidity } \\
\text { and mortality events after } \\
\text { CABG surgery }\end{array}$ & $1 \mathrm{a} / \mathrm{A}$ \\
\hline $\begin{array}{l}\text { Colella et al., [38] } \\
\text { Canada } \\
\text { First-time CABG }\end{array}$ & $\begin{array}{c}\text { RCT } \\
n=124 \\
100 \% \text { male }\end{array}$ & BDI & $\begin{array}{l}\text { At discharge, 6-12 } \\
\text { weeks after }\end{array}$ & - & $\begin{array}{c}\text { Control group: } \\
\text { After: } 8.87(4.74) \\
\text { 6-weeks: } 5.84(5.30) \\
\text { 12-weeks: } 4.43(5.26)\end{array}$ & $\begin{array}{c}\text { Physiological and } \\
\text { psychological challenges } \\
\text { after CABG increases the } \\
\text { depression risk }\end{array}$ & $1 \mathrm{a} / \mathrm{A}$ \\
\hline $\begin{array}{c}\text { Dal Boni et al., [39] } \\
\text { Brazil } \\
\text { Elective CABG }\end{array}$ & $\begin{array}{c}\text { Cross-sectional } \\
n=78 \\
67 \% \text { male }\end{array}$ & BDI & $\begin{array}{l}\text { Before, 2-months } \\
\text { after }\end{array}$ & $8.49(6.87)$ & $5.01(6.61)$ & $\begin{array}{c}\text { CABG had a positive } \\
\text { impact on the patient's } \\
\text { quality of life }\end{array}$ & $2 b / B$ \\
\hline $\begin{array}{c}\text { Doering et al., [40] } \\
\text { USA } \\
\text { First-time CABG }\end{array}$ & $\begin{array}{c}\text { Cohort } \\
n=67 \\
100 \% \text { female }\end{array}$ & HAM-D & $\begin{array}{l}\text { At discharge, } 1 \\
\text { month after }\end{array}$ & - & $41.79 \%$ & $\begin{array}{l}\text { Six months after CABG, } \\
\text { women with major } \\
\text { depression have at } \\
\text { increased risk for infections }\end{array}$ & $2 b / B$ \\
\hline
\end{tabular}


Table 1. Cont

\begin{tabular}{|c|c|c|c|c|c|c|c|}
\hline \multirow{2}{*}{$\begin{array}{c}\text { Study } \\
\text { CABG (First, Elective, Emergency) }\end{array}$} & \multirow{2}{*}{$\begin{array}{l}\text { Design and } \\
\text { Sample }\end{array}$} & \multirow{2}{*}{$\begin{array}{l}\text { Depression } \\
\text { Screening } \\
\text { Instrument }\end{array}$} & \multirow{2}{*}{$\begin{array}{l}\text { Timing of } \\
\text { Assessment }\end{array}$} & \multicolumn{2}{|c|}{ M (SD)/Prevalence } & \multirow{2}{*}{ Main Results } & \multirow{2}{*}{ EL/RG } \\
\hline & & & & Pre & Post & & \\
\hline $\begin{array}{c}\text { Donohue et al., [41] } \\
\text { USA } \\
\text { Elective CABG }\end{array}$ & $\begin{array}{c}\text { RCT } \\
n=2485\end{array}$ & PHQ-2 & At discharge & - & $56 \%$ & $\begin{array}{l}\text { A nurse-guided } \\
\text { intervention in the mental } \\
\text { health area reduces the level } \\
\text { of depression and health } \\
\text { costs post-CABG }\end{array}$ & $1 \mathrm{a} / \mathrm{A}$ \\
\hline $\begin{array}{l}\text { Dunkel et al., [42] } \\
\text { Germany } \\
\text { Elective CABG }\end{array}$ & $\begin{array}{c}\text { Cross-sectional } \\
n=1238 \\
72 \% \text { male }\end{array}$ & PHQ-9 & Before CABG & $21.6 \%$ & - & $\begin{array}{l}\text { Lower age, female gender, } \\
\text { less than } 10 \text { years of } \\
\text { education and living alone } \\
\text { are related to depression } \\
\text { symptoms }\end{array}$ & $2 b / B$ \\
\hline $\begin{array}{l}\text { Dunkel et al., [43] } \\
\text { Germany } \\
\text { Elective CABG }\end{array}$ & $\begin{array}{c}\text { Cross-sectional } \\
n=971 \\
80.1 \% \text { male }\end{array}$ & PHQ-9 & $\begin{array}{c}1-3 \text { days } \\
\text { before, } 1 \text { year after }\end{array}$ & $5.61(4.31)$ & - & $\begin{array}{l}\text { Female gender is related to } \\
\text { depression symptoms } \\
\text { Attributions to stress, } \\
\text { personality and destiny are } \\
\text { associated with higher } \\
\text { depression scores }\end{array}$ & $2 b / B$ \\
\hline $\begin{array}{c}\text { El-Baz et al., [44] } \\
\text { Netherlands and Slovakia } \\
\text { Elective CABG }\end{array}$ & $\begin{array}{c}\text { Observational } \\
\text { multicentre } \\
n \text { total }=226 \\
n 1=114 \text { Slovakia } \\
n 2=112 \\
\begin{array}{c}\text { Netherlands } 80 \% \\
\text { male }\end{array}\end{array}$ & HADS & Before CABG & $\begin{array}{l}n 1=5.01(3.73) \\
n 2=4.96(3.16)\end{array}$ & - & $\begin{array}{l}\text { Female gender, smoking, } \\
\text { lower education, and lower } \\
\text { social support are risks } \\
\text { factors of depression }\end{array}$ & $2 b / B$ \\
\hline $\begin{array}{l}\text { Elliott et al., [45] } \\
\text { Australia } \\
\text { Elective CABG }\end{array}$ & $\begin{array}{c}\text { Cohort } \\
n=174 \\
80 \% \text { male }\end{array}$ & POMS-D & $\begin{array}{l}\text { Before, } 2-6 \text { months } \\
\text { after }\end{array}$ & $10.50(11.76)$ & $\begin{array}{l}\text { 2-months: } 7.38(9.41) \\
\text { 6-months: } 8.32(10.52)\end{array}$ & $\begin{array}{l}\text { The young, male and } \\
\text { smoking are the main risk } \\
\text { factors of depression }\end{array}$ & $2 b / B$ \\
\hline $\begin{array}{c}\text { Feuchtinger et al., [46] } \\
\text { Germany } \\
\text { First-time CABG }\end{array}$ & $\begin{array}{c}\text { Cross-sectional } \\
n=24 \\
37.5 \% \text { male }\end{array}$ & HADS & 1-day before & $\begin{array}{c}6.7(5.1) \\
\text { Low: } 54.17 \% \\
\text { Moderate: } 20.83 \% \\
\text { Severe: } 25 \%\end{array}$ & - & $\begin{array}{l}\text { Interventions such as } \\
\text { information, spiritual } \\
\text { support or cognitive } \\
\text { behavioral therapy are the } \\
\text { key to reduce the feeling of } \\
\text { fear before CABG surgery }\end{array}$ & $2 b / B$ \\
\hline $\begin{array}{c}\text { Freedland et al., [47] } \\
\text { USA } \\
\text { Elective CABG }\end{array}$ & $\begin{array}{c}\text { RCT } \\
n=123 \\
50 \% \text { male }\end{array}$ & $\begin{array}{c}\text { BDI } \\
\text { HAM-D }\end{array}$ & 1 year after & - & $\begin{array}{c}\mathrm{BDI}=22.26(1.3) \\
\mathrm{HAM}-\mathrm{D}=19.53(1)\end{array}$ & $\begin{array}{l}\text { Improvement in perceived } \\
\text { cognitive impairment } \\
\text { correlated with } \\
\text { improvement in depression }\end{array}$ & $1 \mathrm{a} / \mathrm{A}$ \\
\hline $\begin{array}{c}\text { Gallagher \& McKinley, [48] } \\
\text { Australia } \\
\text { Elective CABG }\end{array}$ & $\begin{array}{c}\text { Cohort } \\
n=155 \\
74 \% \text { male }\end{array}$ & HADS & $\begin{array}{c}\text { Before, after } \\
\text { surgery, 2-weeks } \\
\text { after }\end{array}$ & $\begin{array}{c}4.10(3.22) \\
16 \%\end{array}$ & $\begin{array}{l}\text { After: } 18.2 \% \\
4.67(3.49) \\
\text { 2-weeks: } 45 \% \\
6.58(4.03)\end{array}$ & $\begin{array}{l}26.5 \% \text { of patients reported } \\
\text { low perceptions of control } \\
\text { before CABG, } 22 \% \text { after } \\
\text { surgery and } 10.3 \% \text { at } \\
\text { discharge }\end{array}$ & $2 b / B$ \\
\hline
\end{tabular}


Table 1. Cont.

\begin{tabular}{|c|c|c|c|c|c|c|c|}
\hline \multirow{2}{*}{$\begin{array}{c}\text { Study } \\
\text { CABG (First, Elective, Emergency) }\end{array}$} & \multirow{2}{*}{$\begin{array}{l}\text { Design and } \\
\text { Sample }\end{array}$} & \multirow{2}{*}{$\begin{array}{l}\text { Depression } \\
\text { Screening } \\
\text { Instrument }\end{array}$} & \multirow{2}{*}{$\begin{array}{l}\text { Timing of } \\
\text { Assessment }\end{array}$} & \multicolumn{2}{|c|}{ M (SD)/Prevalence } & \multirow{2}{*}{ Main Results } & \multirow{2}{*}{$\mathrm{EL} / \mathrm{RG}$} \\
\hline & & & & Pre & Post & & \\
\hline $\begin{array}{l}\text { Gelogahi et al., [49] } \\
\text { Iran } \\
\text { Elective CABG }\end{array}$ & $\begin{array}{c}\text { RCT } \\
n=40 \\
37.5 \% \text { male }\end{array}$ & DASS & Before, after & $6.67(4.7)$ & $12.1(8.1)$ & $\begin{array}{l}\text { Nurses interventions can } \\
\text { reduce depression levels } \\
\text { after surgery }\end{array}$ & $1 \mathrm{a} / \mathrm{A}$ \\
\hline $\begin{array}{l}\text { Hazavei et al., [50] } \\
\text { Iran } \\
\text { First-time CABG }\end{array}$ & $\begin{array}{c}\text { Quasi-experimental } \\
n=27 \\
77.8 \% \text { male }\end{array}$ & CDS & $\begin{array}{l}\text { Before, } 4-8 \text { weeks } \\
\text { after }\end{array}$ & $104.5(30.4)$ & 2-months after: $89.2(27.8)$ & $\begin{array}{l}\text { Most patients lacked the } \\
\text { skills in health education } \\
\text { and lifestyle-related with } \\
\text { coronary artery disease }\end{array}$ & $1 \mathrm{~b} / \mathrm{A}$ \\
\hline $\begin{array}{l}\text { Horne et al., [51] } \\
\text { Canada } \\
\text { Elective CABG }\end{array}$ & $\begin{array}{l}\text { Cohort } \\
n=104\end{array}$ & PHQ-9 & Before & $60.6 \%$ & - & $\begin{array}{l}\text { Length of stay (more than } 7 \\
\text { days) is associated with a } \\
\text { higher risk of depression }\end{array}$ & $2 b / B$ \\
\hline $\begin{array}{l}\text { Hweidi et al., [52] } \\
\text { Jordan } \\
\text { Elective CABG }\end{array}$ & $\begin{array}{c}\text { Cross-sectional } \\
n=143 \\
53.1 \% \text { male }\end{array}$ & SDS & 2 days after & - & $\begin{array}{l}\text { Mild: } 32.2 \% \\
\text { Moderate: } 60.1 \% \\
\text { Severe: } 5.6 \%\end{array}$ & $\begin{array}{l}\text { Depression is related to } \\
\text { female, unmarried and } \\
\text { unemployed patients }\end{array}$ & $2 b / B$ \\
\hline $\begin{array}{l}\text { Kendel et al., [53] } \\
\text { Germany } \\
\text { Elective CABG }\end{array}$ & $\begin{array}{c}\text { Cohort } \\
n=351 \\
77 \% \text { male }\end{array}$ & PHQ-9 & $\begin{array}{c}2 \text { months, } 2 \text { years } \\
\text { after }\end{array}$ & - & $\begin{array}{c}\text { Male: } 5.38(4.2) \\
\text { Female: } 6.84(4.8)\end{array}$ & $\begin{array}{l}\text { Females have a higher level } \\
\text { of depressive symptoms }\end{array}$ & $2 \mathrm{~b} / \mathrm{B}$ \\
\hline $\begin{array}{l}\text { Kendel et al., [54] } \\
\text { Germany } \\
\text { Elective CABG }\end{array}$ & $\begin{array}{c}\text { Cohort } \\
n=883 \\
80.2 \% \text { male }\end{array}$ & PHQ-9 & $1-3$ days before & $\begin{array}{l}5.38(4.09) \\
8.5 \%\end{array}$ & - & $\begin{array}{l}\text { Depression is related to a } \\
\text { deterioration of physical } \\
\text { condition in patients } \\
\text { undergoing CABG surgery }\end{array}$ & $2 b / B$ \\
\hline $\begin{array}{l}\text { Khoueiry et al., [55] } \\
\text { USA } \\
\text { Elective CABG }\end{array}$ & $\begin{array}{c}\text { Cohort } \\
n=50 \\
56 \% \text { male }\end{array}$ & BDI & $\begin{array}{l}\text { Before, 1-3-6-9 } \\
\text { months follow-up }\end{array}$ & $8 \%$ & $\begin{array}{c}\text { After: } 60 \% \\
\text { 3-months: } 44 \% \\
\text { 6-months: } 40 \% \\
\text { 9-months: } 44 \% \\
\end{array}$ & $\begin{array}{c}\text { Age and gender are not } \\
\text { correlated with depression } \\
\text { levels }\end{array}$ & $2 \mathrm{~b} / \mathrm{B}$ \\
\hline $\begin{array}{l}\text { King et al., [56] } \\
\text { Canada } \\
\text { First-time CABG }\end{array}$ & $\begin{array}{c}\text { Cohort } \\
n=120 \\
100 \% \text { male }\end{array}$ & $\begin{array}{l}\text { BDI } \\
\text { CDS }\end{array}$ & $\begin{array}{l}\text { At discharge, } \\
6-12-36 \text { weeks } \\
\text { follow-up }\end{array}$ & - & $\begin{array}{c}\text { BDI } \\
\text { At discharge: } \\
8.08 \text { (4.76) 4.3\% } \\
\text { 6-weeks: } \\
5.82 \text { (5.36) } 1.9 \% \\
\text { 12-weeks: } \\
4.81 \text { (4.73) 1.9\% } \\
\text { 36-weeks: } \\
4.31 \text { (5.81) 2.1\% } \\
\text { CDS } \\
\text { At discharge: } \\
\text { 74.46 (24.29) 17.2\% } \\
\text { 6-weeks: } \\
\text { 59.58 (25.19) 7.6\% } \\
\text { 12-weeks: } \\
54.56 \text { (23.06) 6.7\% } \\
\text { 36 weeks: } \\
51.22 \text { (23.17) 4.3\% }\end{array}$ & $\begin{array}{l}\text { Family reduces the risk of } \\
\text { depression }\end{array}$ & $2 b / B$ \\
\hline
\end{tabular}


Table 1. Cont.

\begin{tabular}{|c|c|c|c|c|c|c|c|}
\hline \multirow{2}{*}{$\begin{array}{c}\text { Study } \\
\text { CABG (First, Elective, Emergency) }\end{array}$} & \multirow{2}{*}{$\begin{array}{l}\text { Design and } \\
\text { Sample }\end{array}$} & \multirow{2}{*}{$\begin{array}{c}\text { Depression } \\
\text { Screening } \\
\text { Instrument }\end{array}$} & \multirow{2}{*}{$\begin{array}{l}\text { Timing of } \\
\text { Assessment }\end{array}$} & \multicolumn{2}{|c|}{ M (SD)/Prevalence } & \multirow{2}{*}{ Main Results } & \multirow{2}{*}{ EL/RG } \\
\hline & & & & Pre & Post & & \\
\hline $\begin{array}{c}\text { Korbmacher et al., [57] } \\
\text { Germany } \\
\text { Elective CABG }\end{array}$ & $\begin{array}{c}\text { Cohort } \\
n=135 \\
77 \% \text { male }\end{array}$ & HADS & $\begin{array}{c}\text { 1-2 days } \\
\text { before,1-week, } \\
\text { 6-months after }\end{array}$ & $\begin{array}{l}4.3(3.1) \\
20.7 \%\end{array}$ & $\begin{array}{c}\text { 1-week: } \\
5(3.9) 24 \% \\
\text { 6-months: } 4.7(4.3) 28 \%\end{array}$ & $\begin{array}{l}\text { Hight levels of depression } \\
\text { are not associated with } \\
\text { mortality. A } 24.2 \% \text { of } \\
\text { patients with normal scores } \\
\text { before surgery suffers } \\
\text { depression 6-months latter }\end{array}$ & $2 \mathrm{~b} / \mathrm{B}$ \\
\hline $\begin{array}{l}\text { Kozora et al., [58] } \\
\text { USA } \\
\text { Elective CABG }\end{array}$ & $\begin{array}{c}\text { Cohort } \\
n=1156 \\
99.2 \% \text { male }\end{array}$ & BDI & $\begin{array}{l}\text { After, 1-year } \\
\text { follow-up }\end{array}$ & $9.9(7.65)$ & $8.9(7.85)$ & $\begin{array}{l}\text { Older age and lower } \\
\text { education are related to } \\
\text { depression levels }\end{array}$ & $2 b / B$ \\
\hline $\begin{array}{c}\text { Macken et al., [59] } \\
\text { USA } \\
\text { Elective CABG }\end{array}$ & $\begin{array}{c}\text { Quasi-experimental } \\
n=34 \\
76.5 \% \text { male }\end{array}$ & PHQ-9 & After CABG & - & Control group: $18 \%$ & $\begin{array}{l}\text { An intervention cardiac } \\
\text { program can reduce } \\
\text { depressive symptoms }\end{array}$ & $1 \mathrm{~b} / \mathrm{A}$ \\
\hline $\begin{array}{l}\text { McGrady et al., [60] } \\
\text { USA } \\
\text { Elective CABG }\end{array}$ & $\begin{array}{l}\text { Quasi-experimental } \\
\quad n=91\end{array}$ & BDI & After CABG & - & $9.2(7.5)$ & $\begin{array}{c}\text { The symptoms can affect } \\
\text { adherence to prescribed } \\
\text { treatment and may also } \\
\text { affect morbidity and } \\
\text { mortality }\end{array}$ & $1 \mathrm{~b} / \mathrm{A}$ \\
\hline $\begin{array}{c}\text { McKenzie et al., [61] } \\
\text { UK } \\
\text { Elective or emergency CABG }\end{array}$ & $\begin{array}{c}\text { Cross-sectional } \\
n=111 \\
82.9 \% \text { male }\end{array}$ & HADS & After CABG & - & $3.16(3.61) 13.5 \%$ & $\begin{array}{l}\text { Post-operative depression } \\
\text { predicts activities of daily } \\
\text { living functioning }\end{array}$ & $2 b / B$ \\
\hline $\begin{array}{c}\text { McKhann et al., [24] } \\
\text { USA } \\
\text { Elective CABG }\end{array}$ & $\begin{array}{c}\text { Cohort } \\
n=220 \\
73.6 \% \text { male }\end{array}$ & CES-D & $\begin{array}{l}\text { After, } 3 \text { months, } \\
1-3-6 \text { years after }\end{array}$ & - & $\begin{array}{c}\text { Baseline: } 13.2(9.8) 32.4 \% \\
\text { 3-months: } 10.2(9.9) 24.1 \% \\
\text { 1 year: } 9.1(9.8) 17.3 \% \\
\text { 3 year: } 8.9(9.5) 11.8 \% \\
\text { 6 year: } 10.1(9.4) 16.8 \%\end{array}$ & $\begin{array}{l}\text { Depressed patients tended } \\
\text { to have more memory } \\
\text { complaints }\end{array}$ & $2 b / B$ \\
\hline $\begin{array}{l}\text { Modica et al., [62] } \\
\text { Italy } \\
\text { Elective CABG }\end{array}$ & $\begin{array}{c}\text { Cross-sectional } \\
n=1179 \\
80 \% \text { male }\end{array}$ & HADS & After CABG & - & $\begin{array}{c}\text { Moderate-severe: } 10.4 \% \\
\text { Male: } 9.2 \% \\
\text { Female: } 15.4 \%\end{array}$ & $\begin{array}{l}\text { Female gender is related to } \\
\text { a higher depression score }\end{array}$ & $2 \mathrm{~b} / \mathrm{B}$ \\
\hline $\begin{array}{c}\text { Moser et al., [63] } \\
\text { USA } \\
\text { Elective CABG }\end{array}$ & $\begin{array}{c}\text { Observational } \\
\text { multicentre } \\
n=131 \\
94 \% \text { male }\end{array}$ & MAACL & After CABG & - & $\begin{array}{c}13.0(5.5) \\
53 \%\end{array}$ & $\begin{array}{l}\text { Factors such as being a } \\
\text { woman and have lower } \\
\text { educational attainment are } \\
\text { related to depression }\end{array}$ & $2 \mathrm{~b} / \mathrm{B}$ \\
\hline $\begin{array}{l}\text { Murphy et al., [64] } \\
\text { Australia } \\
\text { Elective CABG }\end{array}$ & $\begin{array}{c}\text { Cohort } \\
n=184 \\
79 \% \text { male }\end{array}$ & HADS & $\begin{array}{l}\text { Before, } 2-6 \text { months } \\
\quad \text { follow-up }\end{array}$ & $5.35(4.01)$ & $\begin{array}{l}\text { 2-months: } 4.16(3.71) \\
\text { 6-months: } 3.87(3.51)\end{array}$ & $\begin{array}{l}\text { Over 6-months follow-up } \\
\text { patients show a minor score } \\
\text { of depression }\end{array}$ & $2 \mathrm{~b} / \mathrm{B}$ \\
\hline
\end{tabular}


Table 1. Cont.

\begin{tabular}{|c|c|c|c|c|c|c|c|}
\hline \multirow{2}{*}{$\begin{array}{c}\text { Study } \\
\text { CABG (First, Elective, Emergency) }\end{array}$} & \multirow{2}{*}{$\begin{array}{l}\text { Design and } \\
\text { Sample }\end{array}$} & \multirow{2}{*}{$\begin{array}{l}\text { Depression } \\
\text { Screening } \\
\text { Instrument }\end{array}$} & \multirow{2}{*}{$\begin{array}{l}\text { Timing of } \\
\text { Assessment }\end{array}$} & \multicolumn{2}{|c|}{ M (SD)/Prevalence } & \multirow{2}{*}{ Main Results } & \multirow{2}{*}{$\mathrm{EL} / \mathrm{RG}$} \\
\hline & & & & Pre & Post & & \\
\hline $\begin{array}{l}\text { Nair et al., [65] } \\
\quad \text { India } \\
\text { Elective CABG }\end{array}$ & $\begin{array}{c}\text { Quasi-experimental } \\
n=500 \\
20.2 \% \text { male }\end{array}$ & HADS & 6 months after & - & $20.2 \%$ & $\begin{array}{l}11.6 \% \text { of patients after } \\
\text { CABG adhered to healthy } \\
\text { lifestyle practices }\end{array}$ & $1 \mathrm{~b} / \mathrm{A}$ \\
\hline $\begin{array}{c}\text { Nemati \& Astaneh, [66] } \\
\text { Iran } \\
\text { Elective CABG }\end{array}$ & $\begin{array}{c}\text { Cohort } \\
n=71 \\
73 \% \text { male }\end{array}$ & HADS & $\begin{array}{l}\text { Before, 4-weeks } \\
\quad \text { after }\end{array}$ & $\begin{array}{l}\text { Male: } 13.58(8.54) \\
\text { Female: } 17.88 \\
(7.54)\end{array}$ & $\begin{array}{l}\text { Male: } 9.51(6.00) \\
\text { Female: } 15.05(8.63)\end{array}$ & $\begin{array}{l}\text { CABG surgery can decrease } \\
\text { the level of depression in a } \\
\text { short-term follow-up }\end{array}$ & $2 b / B$ \\
\hline $\begin{array}{c}\text { Nunes et al., [67] } \\
\text { Brazil } \\
\text { Elective CABG }\end{array}$ & $\begin{array}{c}\text { Cohort } \\
n=57 \\
68.42 \% \text { male }\end{array}$ & BDI & $\begin{array}{l}\text { Before, 6-months } \\
\text { after }\end{array}$ & $\begin{array}{c}\text { Minimal: } 56.14 \% \\
\text { Mild: } 26.32 \% \\
\text { Moderate: } 12.28 \% \\
\text { Severe: } 5.26 \%\end{array}$ & $\begin{array}{c}\text { Minimal: } 49.12 \% \\
\text { Mild: } 29.82 \% \\
\text { Moderate: } 17.54 \% \\
\text { Severe: } 3.51 \%\end{array}$ & $\begin{array}{l}\text { Improvement the quality of } \\
\text { life with CABG surgery } \\
\text { reducing depressive } \\
\text { symptoms }\end{array}$ & $2 \mathrm{~b} / \mathrm{B}$ \\
\hline $\begin{array}{l}\text { Okamoto et al., [68] } \\
\text { Japan } \\
\text { Elective or emergency CABG }\end{array}$ & $\begin{array}{c}\text { Cross-sectional } \\
n=79 \\
75.9 \% \text { male }\end{array}$ & HADS & $1-5$ years after & - & $\begin{array}{c}\text { Mild: } 10.1 \% \\
\text { Moderate-severe: } 10.1 \%\end{array}$ & $\begin{array}{l}\text { Depression in CABG } \\
\text { patients is related to a } \\
\text { decrease in functional status } \\
\text { or activities of daily living }\end{array}$ & $2 b / B$ \\
\hline $\begin{array}{l}\text { Oldham et al., [69] } \\
\text { USA } \\
\text { First-time CABG }\end{array}$ & $\begin{array}{c}\text { Cohort } \\
n=131 \\
73 \% \text { male }\end{array}$ & $\begin{array}{l}\text { HAM-D } \\
\text { PHQ-9 } \\
\text { GDS }\end{array}$ & Before & $\begin{array}{l}\text { HAM-D: } 9.9 \% \\
16.3(5.4) \\
\text { PHQ-9: } 56.2 \% \\
13.4(3.9) \\
\text { GDS: } 6.9(3.6)\end{array}$ & - & $\begin{array}{l}\text { Preoperative depression } \\
\text { predicts post-CABG } \\
\text { cognitive health }\end{array}$ & $2 \mathrm{~b} / \mathrm{B}$ \\
\hline $\begin{array}{l}\text { Perrotti et al., [70] } \\
\text { France } \\
\text { Elective CABG }\end{array}$ & $\begin{array}{c}\text { RCT } \\
n=359 \\
85 \% \text { male }\end{array}$ & BDI & Before, 1 year after & $39.6 \%$ & - & $\begin{array}{c}\text { In the first year after CABG, } \\
\text { depressed patients have a } \\
\text { lower improvement and } \\
\text { quality of life }\end{array}$ & $1 \mathrm{a} / \mathrm{A}$ \\
\hline $\begin{array}{l}\text { Perrotti et al., [71] } \\
\text { France } \\
\text { Elective CABG }\end{array}$ & $\begin{array}{c}\text { Cohort } \\
n=272 \\
78 \% \text { male }\end{array}$ & HADS & 2-weeks before & $6 \%$ & - & $\begin{array}{l}\text { CABG surgery improve the } \\
\text { functional mobility, quality } \\
\text { of life and maintenance of } \\
\text { an independent status }\end{array}$ & $2 b / B$ \\
\hline $\begin{array}{c}\text { Phillips-Bute et al., [72] } \\
\text { USA } \\
\text { Elective CABG }\end{array}$ & $\begin{array}{c}\text { Cohort } \\
n=427 \\
70 \% \text { male }\end{array}$ & CES-D & $\begin{array}{l}\text { Before, } 6 \text { months, } 1 \\
\text { year after }\end{array}$ & $\begin{array}{l}\text { Mild-Severe } \\
\text { Male: } 28 \% \\
\text { Female: } 57 \%\end{array}$ & $\begin{array}{c}\text { Mild-severe: } \\
\text { Male: } \\
\text { 6-months: } 17 \% \\
\text { 1-year: } 17 \% \\
\text { Female: } \\
\text { 6-months: } 33 \% \\
\text { 1-year: } 32 \%\end{array}$ & $\begin{array}{l}\text { Depressed patients are more } \\
\text { prone than nondepressed } \\
\text { patients to have a new } \\
\text { cardiac event within } 2 \text { years } \\
\text { of CABG }\end{array}$ & $2 b / B$ \\
\hline $\begin{array}{l}\text { Poole et al., [23] } \\
\text { UK } \\
\text { First-time CABG }\end{array}$ & $\begin{array}{c}\text { Cohort } \\
n=310 \\
86 \% \text { male }\end{array}$ & BDI & $\begin{array}{l}29 \text { days before, } \\
\text { after surgery }\end{array}$ & $\begin{array}{c}8.68(6.61) \\
30.3 \% \\
\text { Minimal: } 69.7 \% \\
\text { Mild: } 25.5 \% \\
\text { Moderate-severe: } \\
4.8 \%\end{array}$ & 8.33 & $\begin{array}{l}\text { Pre-operative depression is } \\
\text { associated with longer } \\
\text { postoperative hospital stays. } \\
\text { The young, female gender, } \\
\text { overweight, smoking and } \\
\text { hypertension variables are } \\
\text { related to depression } \\
\text { symptoms }\end{array}$ & $2 b / B$ \\
\hline
\end{tabular}


Table 1. Cont.

\begin{tabular}{|c|c|c|c|c|c|c|c|}
\hline \multirow{2}{*}{$\begin{array}{c}\text { Study } \\
\text { CABG (First, Elective, Emergency) }\end{array}$} & \multirow{2}{*}{$\begin{array}{l}\text { Design and } \\
\text { Sample }\end{array}$} & \multirow{2}{*}{$\begin{array}{l}\text { Depression } \\
\text { Screening } \\
\text { Instrument }\end{array}$} & \multirow{2}{*}{$\begin{array}{l}\text { Timing of } \\
\text { Assessment }\end{array}$} & \multicolumn{2}{|c|}{ M (SD)/Prevalence } & \multirow{2}{*}{ Main Results } & \multirow{2}{*}{ EL/RG } \\
\hline & & & & Pre & Post & & \\
\hline $\begin{array}{l}\text { Pourafkari et al., [73] } \\
\text { Iran } \\
\text { Elective CABG }\end{array}$ & $\begin{array}{c}\text { Quasi-experimental } \\
n=40 \\
82 \% \text { male }\end{array}$ & BDI & After CABG & - & $\begin{array}{c}25 \% \\
\text { Minimal: } 75 \% \\
\text { Mild: } 12 \% \\
\text { Moderate: } 8 \% \\
\text { Severe: } 5 \%\end{array}$ & $\begin{array}{l}\text { The emergence of } \\
\text { new-onset depression after } \\
\text { CABG is associated with a } \\
\text { poor outcome }\end{array}$ & $1 \mathrm{~b} / \mathrm{A}$ \\
\hline $\begin{array}{l}\text { Rezaei et al., [74] } \\
\text { Iran } \\
\text { Elective CABG }\end{array}$ & $\begin{array}{c}\text { Cohort } \\
n=135 \\
75 \% \text { male }\end{array}$ & SCL-90R & 6 months after & - & $\begin{array}{c}1.17(0.75) \\
44.22 \%\end{array}$ & $\begin{array}{l}\text { The prevalent mental } \\
\text { disorder after CABG is } \\
\text { depression followed by } \\
\text { sensitivity, paranoia, } \\
\text { hostility, anxiety, obsession, } \\
\text { somatization, phobia, and } \\
\text { psychosis }\end{array}$ & $2 b / B$ \\
\hline $\begin{array}{l}\text { Sandau et al., [75] } \\
\text { USA } \\
\text { Elective CABG }\end{array}$ & $\begin{array}{c}\text { Cohort } \\
n=54 \\
78 \% \text { male }\end{array}$ & CES-D & $\begin{array}{l}\text { Before, 3-months } \\
\quad \text { after }\end{array}$ & $\begin{array}{l}14.2(8.6) \\
20 \%\end{array}$ & $10.4(7.5)$ & $\begin{array}{l}\text { Depressive symptoms } \\
\text { remain constant from pre- to } \\
\text { postoperatively at } 3 \text { months }\end{array}$ & $2 \mathrm{~b} / \mathrm{B}$ \\
\hline $\begin{array}{l}\text { Schwarz et al., [76] } \\
\text { Germany } \\
\text { Elective CABG }\end{array}$ & $\begin{array}{c}\text { Cohort } \\
n=47 \\
89 \% \text { male }\end{array}$ & HADS & $\begin{array}{l}\text { Before, 3-months } \\
\quad \text { after }\end{array}$ & $5.0(3.4)$ & $3.8(3.1)$ & $\begin{array}{l}\text { Depression and } \\
\text { health-related quality of life } \\
\text { are not associated with } \\
\text { cognitive dysfunction after } \\
\text { CABG }\end{array}$ & $2 b / B$ \\
\hline $\begin{array}{c}\text { Selnes et al., [25] } \\
\text { USA } \\
\text { Elective or emergency CABG }\end{array}$ & $\begin{array}{c}\text { Cohort } \\
n=152 \\
76 \% \text { male }\end{array}$ & CES-D & $\begin{array}{l}\text { Before, } 12-72 \\
\text { months follow-up }\end{array}$ & $\begin{array}{c}13.2(9.6) \\
33 \%\end{array}$ & $\begin{array}{c}9.5(9.2) \\
13 \%\end{array}$ & $\begin{array}{c}\text { CABG patients had a } \\
\text { decline of score } 72 \text {-months } \\
\text { after }\end{array}$ & $2 \mathrm{~b} / \mathrm{B}$ \\
\hline $\begin{array}{c}\text { Sorensen \& Wang, [77] } \\
\text { USA } \\
\text { First-time CABG }\end{array}$ & $\begin{array}{c}\text { Cohort } \\
n=70 \\
66 \% \text { male }\end{array}$ & GDS & $\begin{array}{l}\text { Before, 6-weeks } \\
\quad \text { after }\end{array}$ & $\begin{array}{c}3.1(2.5) \\
24.2 \%\end{array}$ & $\begin{array}{l}2.4(2.3) \\
15.9 \%\end{array}$ & $\begin{array}{l}\text { Women had greater } \\
\text { depression pre-operative } \\
\text { and post-operative. Length } \\
\text { of stay and age are not } \\
\text { related to depression }\end{array}$ & $2 b / B$ \\
\hline $\begin{array}{c}\text { Spezzaferri et al., [78] } \\
\text { Italy } \\
\text { Elective CABG }\end{array}$ & $\begin{array}{c}\text { Cohort } \\
n=118 \\
100 \% \text { male }\end{array}$ & CBA 2.0-D & $\begin{array}{l}\text { At discharge, } \\
1 \text { year after }\end{array}$ & - & $\begin{array}{l}\text { At discharge: } 12.7 \% \\
\quad 1 \text { year: } 5.9 \%\end{array}$ & $\begin{array}{c}1 \text { year after CABG } \\
\text { depression level decreased }\end{array}$ & $2 \mathrm{~b} / \mathrm{B}$ \\
\hline $\begin{array}{l}\text { Stenman \& Sartipy, [79] } \\
\text { Sweden } \\
\text { Elective and emergency CABG }\end{array}$ & $\begin{array}{l}\text { Cohort } \\
n=302\end{array}$ & PHQ-9 & Before & $29 \%$ & - & $\begin{array}{l}\text { Depressive symptoms are } \\
\text { twice as frequent in women } \\
\text { as in men }\end{array}$ & $2 b / B$ \\
\hline $\begin{array}{l}\text { Thomas et al., [80] } \\
\text { India } \\
\text { First-time CABG }\end{array}$ & $\begin{array}{c}\text { Quasi-experimental } \\
n=100 \\
85 \% \text { male }\end{array}$ & HADS & $\begin{array}{l}\text { Before, 1-week, } 1 \\
\text { month after }\end{array}$ & $4.10(3.30)$ & $\begin{array}{l}\text { 1-week: } 2.03(2.60) \\
\text { 1-month: } 1.26(1.82)\end{array}$ & $\begin{array}{l}\text { Medical adherence behavior } \\
\text { is related to depression six } \\
\text { weeks after surgery }\end{array}$ & $1 \mathrm{~b} / \mathrm{A}$ \\
\hline $\begin{array}{l}\text { Tsai et al., [81] } \\
\text { Taiwan } \\
\text { First-time CABG }\end{array}$ & $\begin{array}{c}\text { Cohort } \\
n=198 \\
81 \% \text { male }\end{array}$ & CSS & $\begin{array}{l}\text { Before, } 1-6 \text { weeks, } \\
3 \text { months } \\
\text { follow-up }\end{array}$ & $2.42(2.64)$ & $\begin{array}{c}\text { 1-week: } 1.41(2.00) \\
\text { 6-weeks: } 1.24(1.86) \\
\text { 3-months: } 0.96(1.70)\end{array}$ & $\begin{array}{l}\text { Age, a longer stay in ICU, } \\
\text { smoking, and lack of } \\
\text { exercise are related to worse } \\
\text { symptoms. } 88 \% \text { of patients } \\
\text { have a trajectory of } \\
\text { depression levels that } \\
\text { decrease over time }\end{array}$ & $2 b / B$ \\
\hline
\end{tabular}


Table 1. Cont.

\begin{tabular}{|c|c|c|c|c|c|c|c|}
\hline \multirow{2}{*}{$\begin{array}{c}\text { Study } \\
\text { CABG (First, Elective, Emergency) }\end{array}$} & \multirow{2}{*}{$\begin{array}{l}\text { Design and } \\
\text { Sample }\end{array}$} & \multirow{2}{*}{$\begin{array}{c}\text { Depression } \\
\text { Screening } \\
\text { Instrument }\end{array}$} & \multirow{2}{*}{$\begin{array}{c}\text { Timing of } \\
\text { Assessment }\end{array}$} & \multicolumn{2}{|c|}{ M (SD)/Prevalence } & \multirow{2}{*}{ Main Results } & \multirow{2}{*}{$\mathrm{EL} / \mathrm{RG}$} \\
\hline & & & & Pre & Post & & \\
\hline $\begin{array}{l}\text { Tully et al., [82] } \\
\text { Australia } \\
\text { First-time CABG }\end{array}$ & $\begin{array}{c}\text { Cohort } \\
n=226 \\
83 \% \text { male }\end{array}$ & DASS & Before, 4 days after & $20.1 \%$ & $23.5 \%$ & $\begin{array}{l}\text { Readmission is related to a } \\
\text { higher depression score. } \\
\text { Depression symptoms are } \\
\text { associated with morbidity }\end{array}$ & $2 b / B$ \\
\hline $\begin{array}{l}\text { Tully et al., [83] } \\
\text { Australia } \\
\text { First-time CABG }\end{array}$ & $\begin{array}{c}\text { Cohort } \\
n=226 \\
83 \% \text { male }\end{array}$ & BDI & $\begin{array}{l}\text { Before, after } \\
\text { surgery }\end{array}$ & $8.62(6.23)$ & $9.05(6.40)$ & $\begin{array}{l}\text { Pessimism, past failure, } \\
\text { self-criticalness and, } \\
\text { worthlessness are } \\
\text { associated with cardiac } \\
\text { morbidity and mortality }\end{array}$ & $2 b / B$ \\
\hline $\begin{array}{l}\text { Yang et al., [84] } \\
\text { China } \\
\text { First-time CABG }\end{array}$ & $\begin{array}{c}\text { Cohort } \\
n=232 \\
81 \% \text { male }\end{array}$ & PHQ-9 & $\begin{array}{l}\text { 3-days before, } \\
\text { 6-months after }\end{array}$ & $\begin{array}{l}4.8(5.0) \\
18.1 \%\end{array}$ & $\begin{array}{l}4.2(5.0) \\
18.1 \%\end{array}$ & $\begin{array}{l}\text { Preoperative depression is } \\
\text { associated with women } \\
\text { gender }\end{array}$ & $2 \mathrm{~b} / \mathrm{B}$ \\
\hline $\begin{array}{c}\text { Yang et al., [85] Taiwan } \\
\text { Elective and emergency CABG }\end{array}$ & $\begin{array}{c}\text { Cross-sectional } \\
n=87 \\
74.7 \% \text { male }\end{array}$ & HADS & $\begin{array}{l}1 \text { week, } 1 \text { month } \\
\text { after }\end{array}$ & - & $\begin{array}{c}\text { 1 week: } \\
\text { Mild: } 17.2 \% \\
\text { Moderate-severe: } 60.9 \% \\
\text { 1 month: } 8.75(4.63) \\
\text { Mild: } 24.1 \% \\
\text { Moderate-severe: } 35.6 \%\end{array}$ & $\begin{array}{l}\text { Depression is related to } \\
\text { sleep quality after CABG } \\
\text { surgery }\end{array}$ & $2 \mathrm{~b} / \mathrm{B}$ \\
\hline $\begin{array}{c}\text { Yüksel et al., [86] } \\
\text { Turkey } \\
\text { Elective and first-CABG }\end{array}$ & $\begin{array}{c}\text { Cohort } \\
n=63 \\
\text { G1: diagnosed } \\
\text { after } \\
\text { experiencing an } \\
\text { ACS } \\
\text { G2: diagnosed } \\
\text { without } \\
\text { an ACS }\end{array}$ & BDI & Before & $\begin{array}{c}\text { G1:14.9 (9.5) } \\
\text { G2: } 12.1(7.4) \\
66.6 \% \\
\text { Mild: } 22.2 \% \\
\text { Moderate-severe: } \\
44.4 \%\end{array}$ & - & $\begin{array}{l}\text { Patients in both groups } \\
\text { were found to be depressed } \\
\text { and hopeless about the } \\
\text { future }\end{array}$ & $2 \mathrm{~b} / \mathrm{B}$ \\
\hline $\begin{array}{c}\text { Zimmerman et al., [87] } \\
\text { USA } \\
\text { Elective CABG }\end{array}$ & $\begin{array}{c}\text { RCT } \\
n=226 \\
83 \% \text { male }\end{array}$ & CSS & At discharge & - & $19 \%$ & $\begin{array}{l}\text { Health care providers must } \\
\text { assist the patients before } \\
\text { hospital discharge to } \\
\text { identify the risks and } \\
\text { difficulties in patients after } \\
\text { CABG up to } 6 \text { months after } \\
\text { surgery }\end{array}$ & $1 \mathrm{a} / \mathrm{A}$ \\
\hline
\end{tabular}

ACS = Acute Coronary Syndrome; BDI = Beck Depression Inventory; CABG = Coronary Artery Bypass Graft; CBA 2.0-D = Depression scales of the Cognitive Behavioural Assessment; CDS = Cardiac Depression Scale; CES-D = Center for Epidemiological Studies Depression Scale; CSS = Cardiac Symptom Survey; DASS = Depression, Anxiety, Stress scale; GDS = Geriatric Depression Scale; HADS = Hospital Anxiety and Depression Scale; HAM-D = Hamilton Rating Scale for Depression; ICU = Intensive care unit; MAACL = Multiple Affect Adjective Checklist; PHQ-2 = Patient Health Questionnaire 2-item; PHQ-9 = Patient Health Questionnaire 9-item; POMS-D = Profile of Mood State Depression Scale; RCT = Randomized Clinical Trial; SDS = Self-rating Depression Scale; SCL-90R = Symptom Checklist-90 Revised. 


\subsection{Measurement of Depression}

We used a total of 15 measurement tools. The Hospital Anxiety and Depression Scale (HADS) $(n=18)$, Beck Depression Inventory (BDI) $(n=17)$, nine-item Patient Health Questionnaire (PHQ-9) $(n=9)$, and Centre for Epidemiological Studies Depression Scale (CES-D) $(n=4)$ were the measurement tools used (Table 1 and Supplementary Table S1).

\subsection{Meta-Analysis}

A total of 1217 patients were included in the meta-analysis prior to CABG surgery, and 596 patients after the operation. Egger's test showed no publication bias in any case.

For the HADS tool, the prevalence of depression prior to surgery $(n=144)$ was $19 \%(95 \% \mathrm{CI}=9-31)$ with a high degree of heterogeneity $\left(\mathrm{I}^{2}=93.4 \%\right)$, while the prevalence after surgery $(n=394)$ was $19 \%$ $(95 \% \mathrm{CI}=13-26)$ with $\mathrm{I}^{2}=92.2 \%$, according to the random effects model (Figures 2 and 3 ).

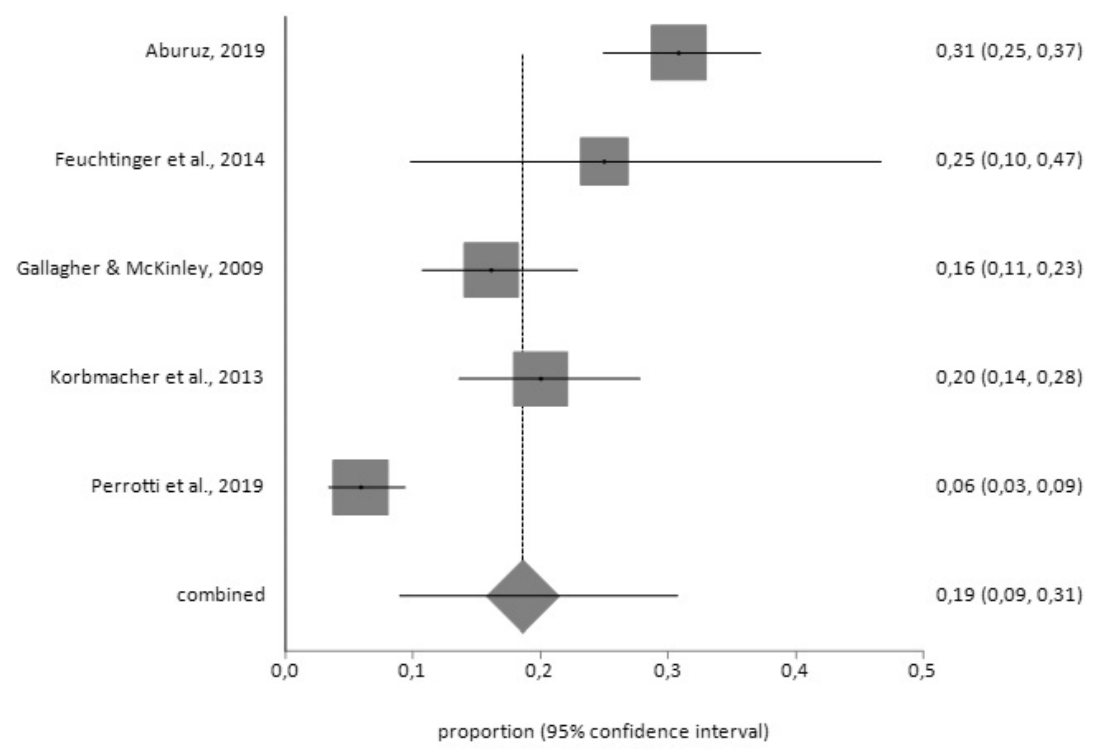

Figure 2. Forest plot for pre-CABG depression using Hospital Anxiety and Depression Scale (HADS).

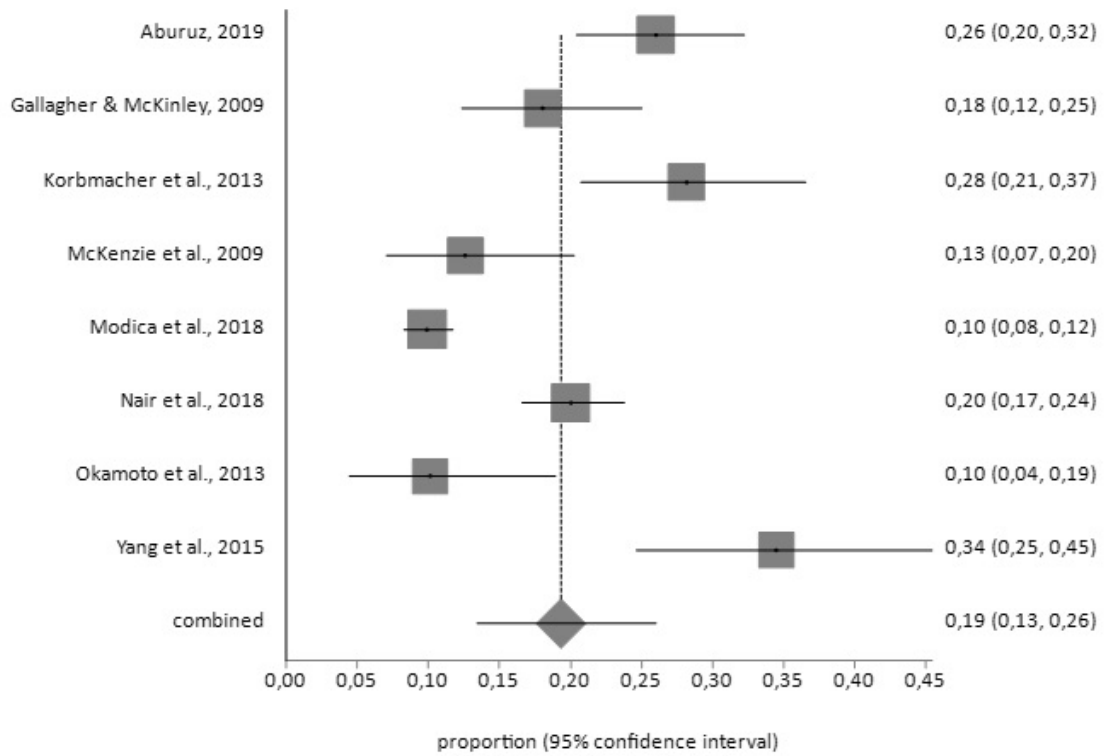

Figure 3. Forest plot for post-CABG depression using HADS. 
For the BDI tool, the prevalence of depression prior to surgery $(n=469)$ was $37 \%(95 \% \mathrm{CI}=28-46)$ with a high degree of heterogeneity $\left(\mathrm{I}^{2}=89.4 \%\right)$, while the prevalence afterwards $(n=97)$ was $33 \%$ $(95 \% \mathrm{CI}=12-59)$ with a high degree of heterogeneity $\left(\mathrm{I}^{2}=96.6 \%\right)$, according to the random effects model (Figures 4 and 5).

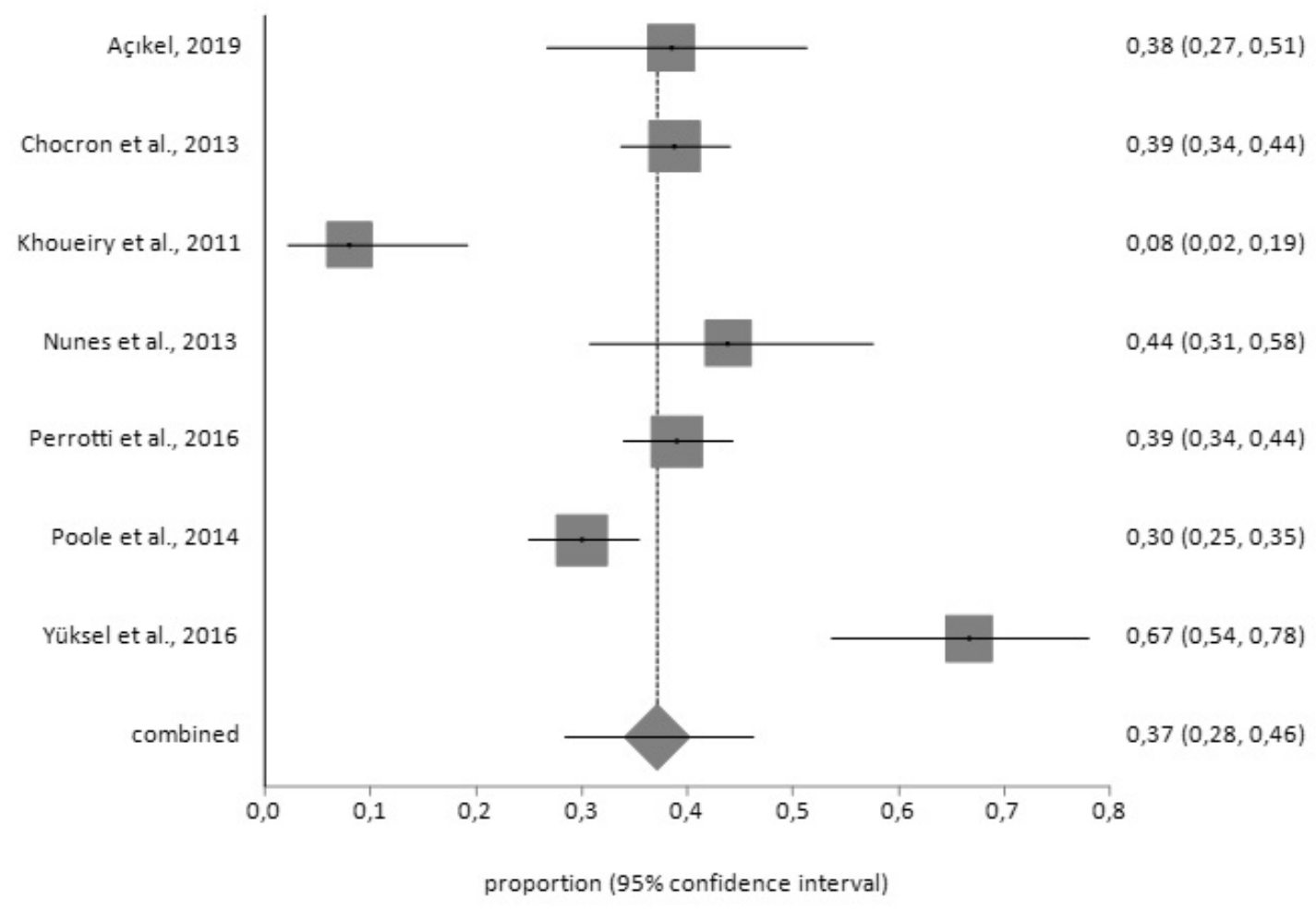

Figure 4. Forest plot for pre-CABG depression using Beck Depression Inventory (BDI).

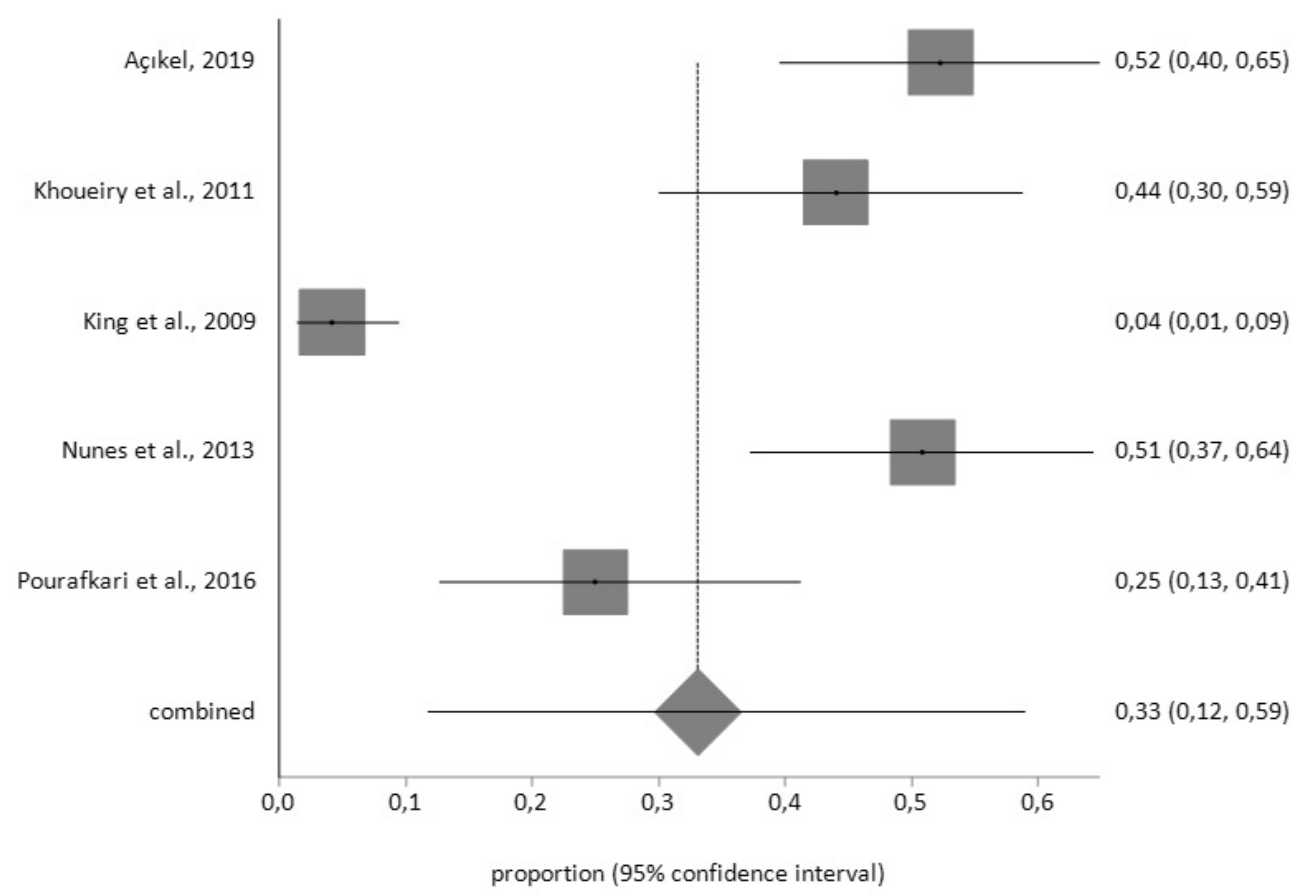

Figure 5. Forest plot for post-CABG depression using BDI. 
According to the PHQ-9 tool, the prevalence prior to surgery $(n=543)$ was $22 \%(95 \% \mathrm{CI}=12-33)$ with a high degree of heterogeneity $\left(\mathrm{I}^{2}=97.5 \%\right)$ according to the random effects model; and, the prevalence of depression after surgery $(n=48)$, using the fixed effects model, was $18 \%(95 \% \mathrm{CI}=14-23)$ (Figures 6 and 7), with a low degree of heterogeneity $\left(\mathrm{I}^{2}=2 \%\right)$.

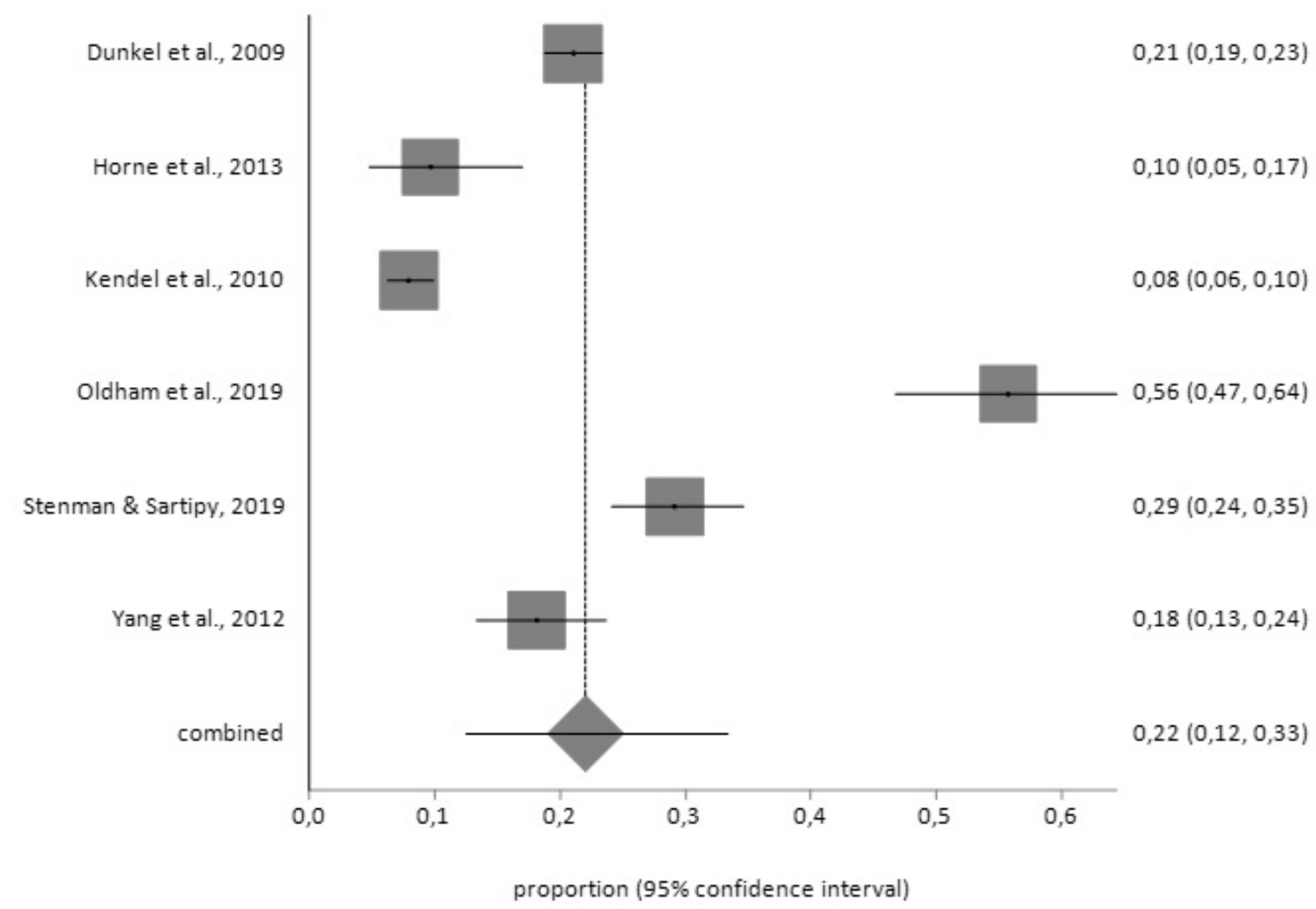

Figure 6. Forest plot for pre-CABG depression using Patient Health Questionnaire (PHQ-9).

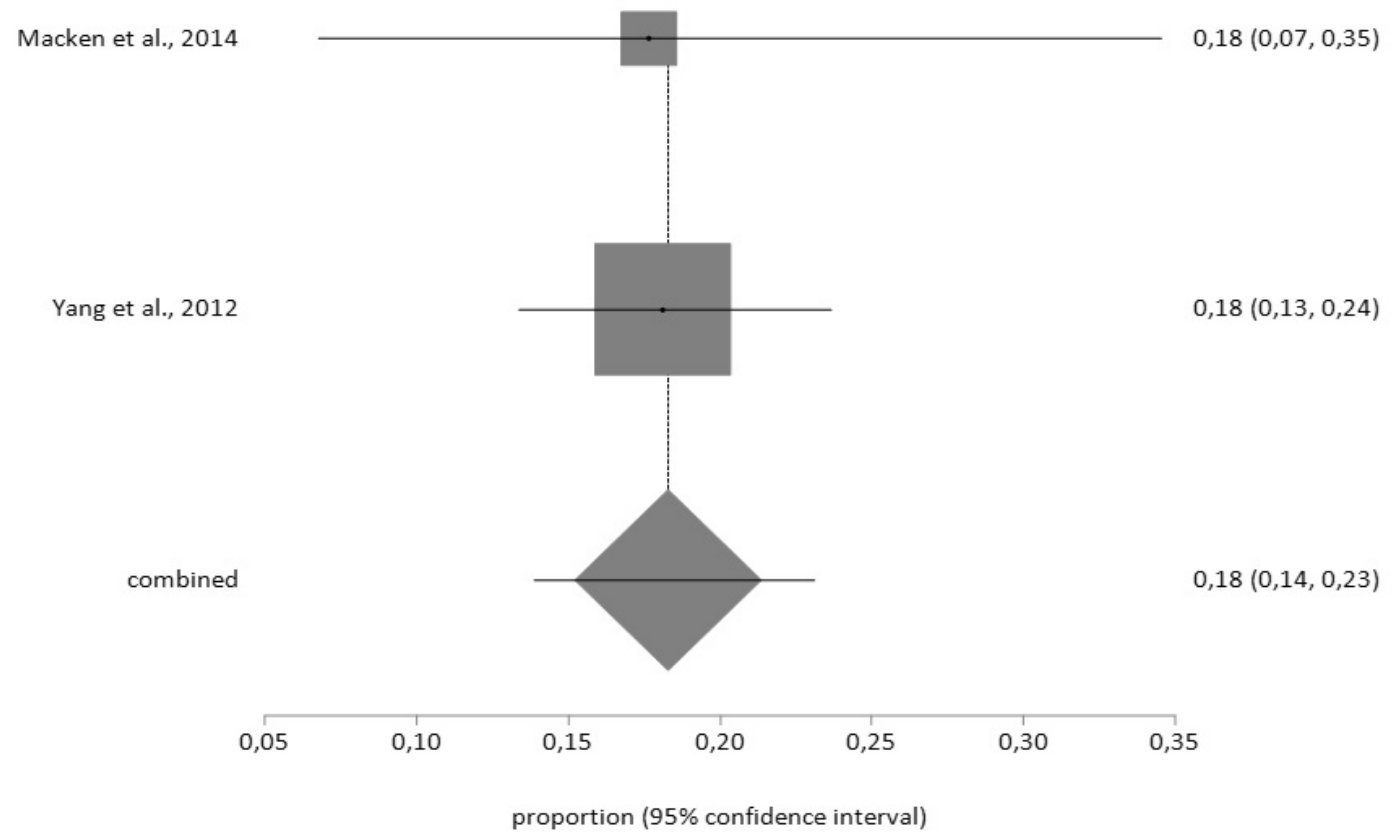

Figure 7. Forest plot for post-CABG depression using PHQ-9. 
Finally, for CES-D, the prevalence of pre-CABG depression $(n=61)$ using the random effects model was $28 \%(95 \% \mathrm{CI}=17-40)$ with a moderate degree of heterogeneity $\left(\mathrm{I}^{2}=66.9 \%\right)$; while the prevalence after surgery $(n=57)$ was $15 \%(95 \% C I=12-19)$ with a low degree of heterogeneity $\left(\mathrm{I}^{2}=2 \%\right)$, according to the fixed effects model (Figures 8 and 9 )

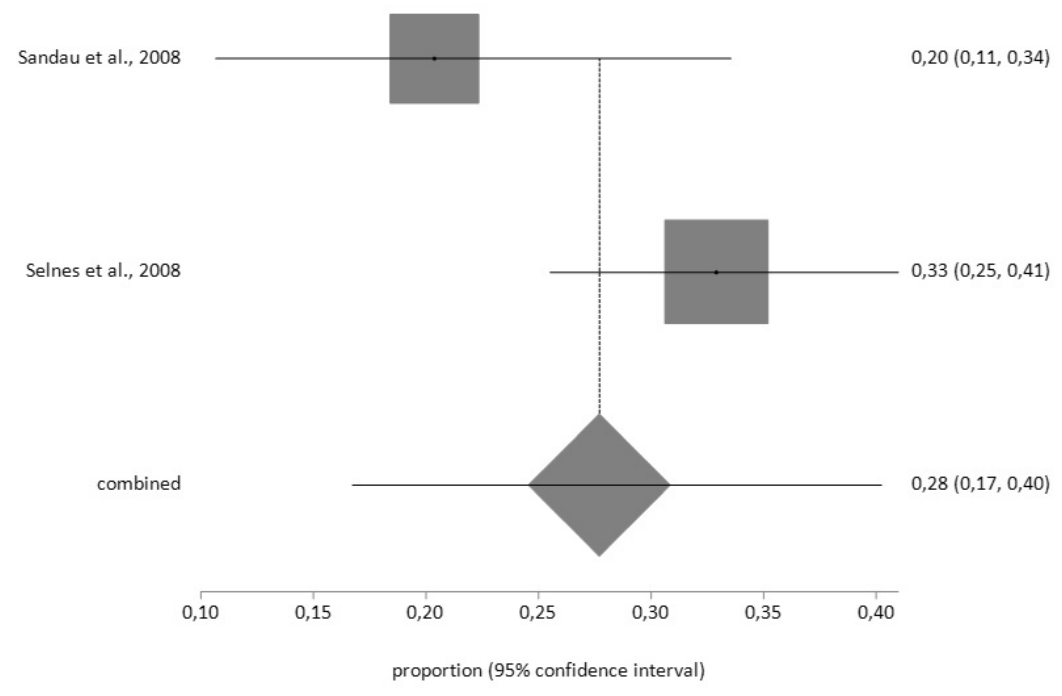

Figure 8. Forest plot for pre-CABG depression using Centre for Epidemiological Studies Depression Scale (CES-D).

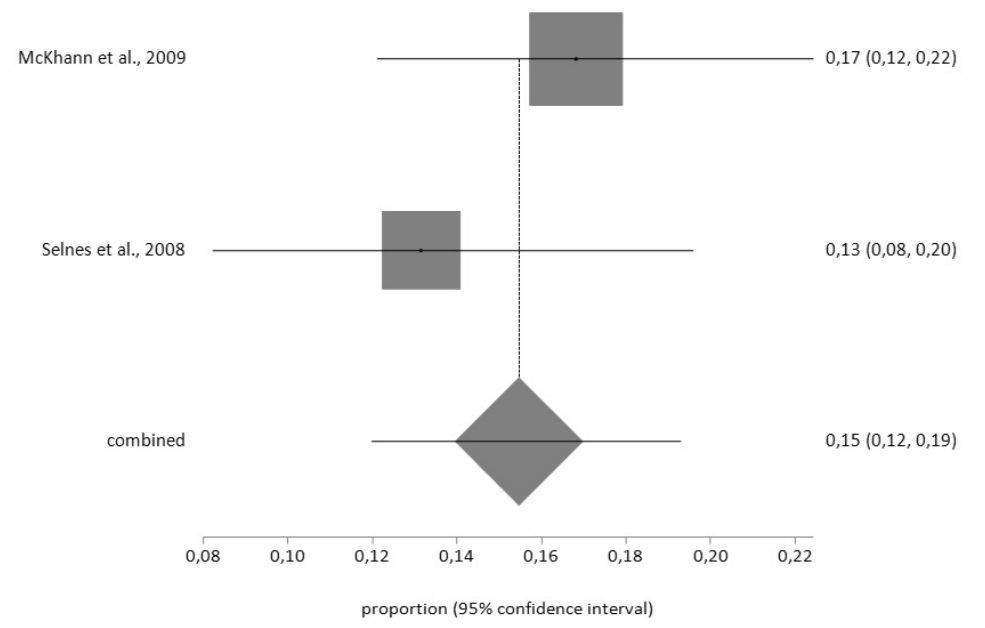

Figure 9. Forest plot for post-CABG depression using CES-D.

\subsection{Levels of Depression Before and After CABG Surgery and Follow Up}

Prior to $C A B G$ surgery, most of the authors report depression levels within the normal range, although others found mild $[36,55,58,83,86]$ and moderate levels $[27,35,66,69]$ (Table 1).

Post-surgery, most authors report normal levels, while others found mild $[28,36,49,53,58,60,66,83,85,88]$, moderate $[27,47,69]$, and severe [32] levels.

The majority of authors observed a positive impact on depression prevalence and levels after surgery, as well as in the short and medium term, although others found that these levels increased after surgery $[28,32,33,48,49,55,57,89]$.

\section{Discussion}

The prevalence of depression obtained in this study varied between $19 \%$ and $37 \%$ prior to surgery, and between $15 \%$ and $33 \%$ after surgery, depending on the type of measurement tool 
used. Other studies that combine CABG with valve replacement have reported similar percentages, with depression prevalence ranging from $15 \%$ pre-CABG [90] to $37.7 \%$ post-CABG [51,91], associated with the development of the disease, worse quality of life, longer hospital stays, and high rates of hospital readmissions [8].

Normal levels of pre-CABG depression are observed, although other studies have indicated higher levels, from moderate to severe [92]. However, more than $25 \%$ of patients with normal levels are at risk of worsening, for which reason continuous reassessment can identify patients with transient symptoms of depression [93].

High levels of depression prior to the operation predict a worse quality of life [94,95], worse survival after a CABG [12,96], and more symptoms up to six months after surgery [97].

We have observed that depression levels did not go to remission, but they tend to improve in depressive symptoms, which is probably due to an improvement in the patient's quality of life [98], and even due to greater optimism that facilitates commitment to adaptation [99]. Some authors have found a positive impact on patients from eight weeks [100], while others report a slight improvement from the first month post-CABG surgery [101]. For the majority of patients, depression persists after the surgery. Recent meta-analyses demonstrated that patients undergoing heart valve surgery are at risk of cognitive dysfunction up to six months after surgery [102,103].

Although there is a relationship between depression and CABG, its temporal onset is not clear. Depression can be a pre-existing condition, which increases the risk of cardiovascular disease that is related to behavioural alterations in diet, physical activity level, toxic habits, or poor adherence to treatment and recommendations [45]; or, can appear as a consequence of multiple postoperative complications, such as longer hospital stays [23], readmissions [104,105], general pain [104], or even when facing a series of lifestyle changes [12].

Without evaluation, it is unlikely that depression is being treated correctly. Some authors report that more than $50 \%$ of patients were receiving medical treatment for depression, even though they had no symptoms of depression [106]. For this reason, the use of measurement tools to confirm the presence and levels of depression makes it possible to identify the at-risk patients, and therefore carry out a more in-depth post-CABG follow-up, of at least nine months [93].

The current study highlights the importance of depression measures before and after CABG in assessing clinically meaningful mood disturbance, in order to provide early intervention. Systematic screening for depression in the period both before and after this procedure is crucial. Planned coaching combined with counselling can reduce these levels [36]. Cardiac rehabilitation programmes $[107,108]$ and cognitive-behavioural therapies are also available, which reduce the levels of depression and even decrease the length of hospital stays [109]. However, further studies are needed to understand the potential prognostic implication of depression and investigate the best ways to approach the treatment of depression in this patient group.

Depression counselling prior to surgery can influence the post-surgical depression levels by positively improving a patient's perception of illness control and management [13]. Planning is therefore an essential part of the healthcare process as it has the potential to promote self-care [36].

From a clinical perspective, these results suggest that strategies that are aimed to improve depression as a disorder, such as the application of policies and depression assessment protocols prior to CABG by health care providers, are essential, because the depression level might help risk stratification in patients undergoing CABG identifying the high-risk groups and the trajectory of recovery experienced [11].

This study has several limitations. Firstly, the heterogeneity in terms of prevalence is due to different estimation methods over time, differences in the timing of assessment and demographic differences between samples, different uses of cut-offs on questionnaire measures, as well as the use of various tools for assessing the symptoms of depression. Secondly, the measuring tools assess the severity of depression symptoms, but they do not replace a formal clinical diagnosis of depression. 


\section{Conclusions}

There is a high presence of depression both before and after CABG surgery. While this study found an overall improvement in depressive symptoms after CABG, depression persists after the surgery for the majority of patients. The depression levels present prior to the operation may affect postoperative recovery.

Given the prevalence of depression and its impact, early detection is crucial, since it enables the identification of at-risk patients, through a clinical interview that uses validated measurement tools. This enables the medical team to implement preventive strategies as well as monitor the development of the depression.

Supplementary Materials: The following are available online at http://www.mdpi.com/2077-0383/9/4/909/s1, Table S1: Depression Assessment Instruments Used by the 65 Studies Included in the Systematic Review [110-122].

Author Contributions: J.S.-R., J.L.G.-U. and M.C.-R. conceived and designed the review and meta-analysis; M.A.E., N.S.-M. and M.J.M.-J. contributed to the selection process and analysis of the risk of bias; N.S.-M. and J.L.G.-U. contributed to the meta-analysis; J.S.-R., M.C.-R. and A.V.-S. contributed to the data extraction; N.S.-M. and M.C.-R. wrote the paper; J.S.-R. and J.L.G.-U. drafted the paper. All authors have read and agreed to the published version of the manuscript.

Funding: This research received no external funding.

Acknowledgments: The results reported in the study are from the doctoral thesis of Moath Abu Ejheisheh and belong to the Clinical Medicine and Health Public Programme (B 12.56.1) of the University of Granada, Spain.

Conflicts of Interest: The authors declare no conflict of interest.

\section{References}

1. Le, J.; Dorstyn, D.S.; Mpfou, E.; Prior, E.; Tully, P.J. Health-related quality of life in coronary heart disease: A systematic review and meta-analysis mapped against the International Classification of Functioning, Disability and Health. Qual. Life Res. 2018, 27, 2491-2503. [CrossRef] [PubMed]

2. Alexander, J.H.; Smith, P.K. Coronary-Artery Bypass Grafting. N. Engl. J. Med. 2016, 374, 1954-1964. [CrossRef] [PubMed]

3. Noyez, L.; de Jager, M.J.; Markou, A.L.P. Quality of life after cardiac surgery: Underresearched research. Interact. Cardiovasc. Thorac. Surg. 2011, 13, 511-515. [CrossRef] [PubMed]

4. Karim, M.N.; Reid, C.M.; Huq, M.; Brilleman, S.L.; Cochrane, A.; Tran, L.; Billah, B. Predicting long-term survival after coronary artery bypass graft surgery. Interact. Cardiovasc. Thorac. Surg. 2018, 26, 257-263. [CrossRef] [PubMed]

5. AbuRuz, M.E.; Al-Dweik, G.; Al-Akash, H.Y. Checking the moderating effect of perceived control on the relationship between anxiety and postoperative hospital length of stay among coronary artery bypass graft patients. Int. J. Gen. Med. 2019, 12, 79-85. [CrossRef] [PubMed]

6. Kidd, T.; Poole, L.; Ronaldson, A.; Leigh, E.; Jahangiri, M.; Steptoe, A. Attachment anxiety predicts depression and anxiety symptoms following coronary artery bypass graft surgery. Br. J. Health Psychol. 2016, 21, 796-811. [CrossRef]

7. Celano, C.M.; Huffman, J.C. Depression and cardiac disease: A review. Cardiol. Rev. 2011, 19, 130-142. [CrossRef]

8. Iribarne, A.; Chang, H.; Alexander, J.H.; Gillinov, A.M.; Moquete, E.; Puskas, J.D.; Bagiella, E.; Acker, M.A.; Mayer, M.L.; Ferguson, T.B.; et al. Readmissions after cardiac surgery: Experience of the national institutes of health/canadian institutes of health research cardiothoracic surgical trials network. Ann. Thorac. Surg. 2014, 98, 1274-1280. [CrossRef]

9. Beck, A.; Steer, R. Manual for the Beck Depression Inventory, 1993 ed.; Psychological Corporation: San Antonio, TX, USA, 1987.

10. Zigmond, A.S.; Snaith, R.P. The hospital anxiety and depression scale. Acta Psychiatr. Scand. 1983, 67, 361-370. [CrossRef]

11. Ravven, S.; Bader, C.; Azar, A.; Rudolph, J.L. Depressive symptoms after CABG surgery: A meta-analysis. Harv. Rev. Psychiatry 2013, 21, 59-69. [CrossRef] 
12. Stenman, M.; Holzmann, M.J.; Sartipy, U. Association between preoperative depression and long-term survival following coronary artery bypass surgery-A systematic review and meta-analysis. Int. J. Cardiol. 2016, 222, 462-466. [CrossRef] [PubMed]

13. Rushton, M.; Howarth, M.; Grant, M.J.; Astin, F. Person-centred discharge education following coronary artery bypass graft: A critical review. J. Clin. Nurs. 2017, 26, 5206-5215. [CrossRef] [PubMed]

14. Abdulrahman, M.; Alsabbagh, A.; Kuntze, T.; Lauer, B.; Ohlow, M.A. Impact of hierarchy on multidisciplinary heart-team recommendations in patients with isolated multivessel coronary artery disease. J. Clin. Med. 2019, 8, 1490. [CrossRef]

15. McKenzie, L.; Simpson, J.; Stewart, M. A systematic review of pre-operative predictors of post-operative depression and anxiety in individuals who have undergone coronary artery bypass graft surgery. Psychol. Health Med. 2010, 15, 74-93. [CrossRef]

16. Protogerou, C.; Fleeman, N.; Dwan, K.; Richardson, M.; Dundar, Y.; Hagger, M.S. Moderators of the effect of psychological interventions on depression and anxiety in cardiac surgery patients: A systematic review and meta-analysis. Behav. Res. Ther. 2015, 73, 151-164. [CrossRef] [PubMed]

17. Keeping-Burke, L.; Purden, M.; Frasure-Smith, N.; Cossette, S.; McCarthy, F.; Amsel, R. Bridging the transition from hospital to home: Effects of the VITAL telehealth program on recovery for CABG surgery patients and their caregivers. Res. Nurs. Health 2013, 36, 540-553. [CrossRef]

18. Moher, D.; Shamseer, L.; Clarke, M.; Ghersi, D.; Liberati, A.; Petticrew, M.; Shekelle, P.; Stewart, L. Preferred reporting items for systematic review and meta-analysis protocols (PRISMA-P) 2015 statement. Syst. Rev. 2015, 4, 1. [CrossRef] [PubMed]

19. Sanderson, S.; Tatt, I.D.; Higgins, J.P.T. Tools for assessing quality and susceptibility to bias in observational studies in epidemiology: A systematic review and annotated bibliography. Int. J. Epidemiol. 2007, 36, 666-676. [CrossRef]

20. Higgins, J.P.T.; Green, S. Cochrane Handbook for Systematic Reviews of Interventions. Version 5.1.0. The Cochrane Collaboration. Available online: www.cochrane-handbook.org (accessed on 10 September 2019).

21. Howick, J.; Chalmers, I.; Glasziou, P.; Greenhalg, T.; Heneghan, C.; Liberati, A.; Moschetti, I.; Phillips, B.; Thornton, H. The Oxford 2011 Levels of Evidence. Available online: https://www.cebm.net/2016/05/ocebmlevels-of-evidence (accessed on 10 September 2019).

22. Higgins, J.P.T.; Thompson, S.G.; Deeks, J.J.; Altman, D.G. Measuring inconsistency in meta-analyses. Br. Med. J. 2003, 327, 557-560. [CrossRef]

23. Poole, L.; Leigh, E.; Kidd, T.; Ronaldson, A.; Jahangiri, M.; Steptoe, A. The combined association of depression and socioeconomic status with length of post-operative hospital stay following coronary artery bypass graft surgery: Data from a prospective cohort study. J. Psychosom. Res. 2014, 76, 34-40. [CrossRef]

24. McKhann, G.M.; Selnes, O.A.; Grega, M.A.; Bailey, M.M.; Pham, L.D.; Baumgartner, W.A.; Zeger, S.L. Subjective memory symptoms in surgical and nonsurgical coronary artery patients: 6-year follow-up. Ann. Thorac. Surg. 2009, 87, 27-34. [CrossRef] [PubMed]

25. Selnes, O.A.; Grega, M.A.; Bailey, M.M.; Pham, L.D.; Zeger, S.L.; Baumgartner, W.A.; McKhann, G.M. Cognition 6 years after surgical or medical therapy for coronary artery disease. Ann. Neurol. 2008, 63, 581-590. [CrossRef] [PubMed]

26. Abbott, A.; Barnason, S.; Zimmerman, L. Symptom burden clusters and their impact on psychosocial functioning following coronary artery bypass surgery. J. Cardiovasc. Nurs. 2010, 25, 301-310. [CrossRef] [PubMed]

27. Aburuz, M.E. Pre-operative depression predicted longer hospital length of stay among patients undergoing coronary artery bypass graft surgery. Risk Manag. Healthc. Policy 2019, 12, 75-83. [CrossRef] [PubMed]

28. Açıkel, M.E.T. Evaluation of depression and anxiety in coronary artery bypass surgery patients: A prospective clinical study. Braz. J. Cardiovasc. Surg. 2019, 34, 389-395. [CrossRef] [PubMed]

29. Afridi, M.I.; Habib, S.; Lal, C.; Khan, A.U.R.; Afaq, S.M.; Tariq, Q.D. Frequency of depression in patients undergoing coronary artery bypass grafting surgery (CABGS), before the surgery, at discharge and at six months follow up. J. Liaquat Univ. Med. Heal. Sci. 2016, 15, 110-115.

30. Ajtahed, S.S.; Rezapour, T.; Etemadi, S.; Moradi, H.; Habibi Asgarabad, M.; Ekhtiari, H. Efficacy of neurocognitive rehabilitation after coronary artery bypass graft surgery in improving quality of life: An interventional trial. Front. Psychol. 2019, 10, 1759. [CrossRef] 
31. Ammouri, A.A.; Al-Daakak, Z.M.; Gharaibeh, H.; Al-Zaru, I. Symptoms experienced by jordanian men and women after coronary artery bypass graft surgery. Dimens. Crit. Care Nurs. 2016, 35, 125-132. [CrossRef]

32. Amouzeshi, A.; Hosseini, S.M.; Javadi, A.; Norozian, F.; Zamanian, N.; Yahya, M.; Jomefourjan, S.; Amouzeshi, Z.; Kohan, M. Depression and associated factors in patients undergoing coronary artery bypass grafting in Imam Reza Hospital of Mashhad in 2013. J. Surg. Trauma 2015, 3, 51-56.

33. Azzopardi, S.; Lee, G. Health-related quality of life 2 years after coronary artery bypass graft surgery. J. Cardiovasc. Nurs. 2009, 24, 232-240. [CrossRef]

34. Bay, P.S.; Beckman, D.; Trippi, J.; Gunderman, R.; Terry, C. The effect of pastoral care services on anxiety, depression, hope, religious coping, and religious problem solving styles: A randomized controlled study. J. Relig. Health 2008, 47, 57-69. [CrossRef] [PubMed]

35. Beresnevaite, M.; Benetis, R.; Taylor, G.J.; Jurnien, K.; Kinduris, Š.; Barauskien, V. Depression predicts perioperative outcomes following coronary artery bypass graft surgery. Scand. Cardiovasc. J. 2010, 44, 289-294. [CrossRef] [PubMed]

36. Cebeci, F.; Çelik, S.Ş. Effects of discharge teaching and counselling on anxiety and depression level of CABG patients. Turk. J. Thorac. Cardiovasc. Surg. 2011, 19, 170-176. [CrossRef]

37. Chocron, S.; Vandel, P.; Durst, C.; Laluc, F.; Kaili, D.; Chocron, M.; Etievent, J.-P. Antidepressant therapy in patients undergoing coronary artery bypass grafting: The MOTIV-CABG trial. Ann. Thorac. Surg. 2013, 95, 1609-1618. [CrossRef]

38. Colella, T.J.; King-Shier, K. The effect of a peer support intervention on early recovery outcomes in men recovering from coronary bypass surgery: A randomized controlled trial. Eur. J. Cardiovasc. Nurs. 2018, 17, 408-417. [CrossRef] [PubMed]

39. Dal Boni, A.L.M.; Martinez, J.E.; Da Silva Saccomann, I.C.R. Quality of Life of patients undergoing coronary artery bypass grafting. Acta Paul. Enferm. 2013, 26, 575-580. [CrossRef]

40. Doering, L.V.; Martínez-Maza, O.; Vredevoe, D.L.; Cowan, M.J. Relation of depression, natural killer cell function, and infections after coronary artery bypass in women. Eur. J. Cardiovasc. Nurs. 2008, 7, 52-58. [CrossRef]

41. Donohue, J.M.; Belnap, B.H.; Men, A.; He, F.; Roberts, M.S.; Schulberg, H.C.; Reynolds, C.F.; Rollman, B.L. Twelve-month cost-effectiveness of telephone-delivered collaborative care for treating depression following CABG surgery: A randomized controlled trial. Gen. Hosp. Psychiatry 2014, 36, 453-459. [CrossRef]

42. Dunkel, A.; Kendel, F.; Lehmkuhl, E.; Babitsch, B.; Oertelt-Prigione, S.; Hetzer, R.; Regitz-Zagrosek, V. Predictors of preoperative depressive risk in patients undergoing coronary artery bypass graft surgery. Clin. Res. Cardiol. 2009, 98, 643-650. [CrossRef]

43. Dunkel, A.; Kendel, F.; Lehmkuhl, E.; Hetzer, R.; Regitz-Zagrosek, V. Causal attributions among patients undergoing coronary artery bypass surgery: Gender aspects and relation to depressive symptomatology. J. Behav. Med. 2011, 34, 351-359. [CrossRef]

44. El-Baz, N.; Ondusova, D.; Studencan, M.; Rosenberger, J.; Reijneveld, S.A.; van Dijk, J.P.; Middel, B. Differences between Slovak and Dutch patients scheduled for coronary artery bypass graft surgery regarding clinical and psychosocial predictors of physical and mental health-related quality of life. Eur. J. Cardiovasc. Nurs. 2018, 17, 324-335. [CrossRef] [PubMed]

45. Elliott, P.C.; Murphy, B.M.; Oster, K.A.; Le Grande, M.R.; Higgins, R.O.; Worcester, M.U.C. Changes in mood states after coronary artery bypass graft surgery. Eur. J. Cardiovasc. Nurs. 2010, 9, 188-194. [CrossRef] [PubMed]

46. Feuchtinger, J.; Burbaum, C.; Heilmann, C.; Imbery, C.; Siepe, M.; Stotz, U.; Fritzsche, K.; Beyersdorf, F. Anxiety and fear in patients with short waiting times before coronary artery bypass surgery-A qualitative study. J. Clin. Nurs. 2014, 23, 1900-1907. [CrossRef] [PubMed]

47. Freedland, K.; Skala, J.; Carney, R.; Rubin, E.; Lustman, P.; Dávila-Román, V.; Steinmeyer, B.; Hogue, C. Treatment of depression after coronary artery bypass surgery: A randomized controlled trial. Arch. Gen. Psychiatry 2009, 66, 387-396. [CrossRef] [PubMed]

48. Gallagher, R.; McKinley, S. Anxiety, depression and perceived control in patients having coronary artery bypass grafts. J. Adv. Nurs. 2009, 65, 2386-2396. [CrossRef] [PubMed]

49. Gelogahi, Z.K.; Aghebati, N.; Mazloum, S.R.; Mohajer, S. Effectiveness of nurse's intentional presence as a holistic modality on depression, anxiety, and stress of cardiac surgery patients. Holist. Nurs. Pract. 2018, 32, 296-306. [CrossRef] 
50. Hazavei, S.M.M.; Sabzmakan, L.; Hasanzadeh, A.; Rabiei, K.; Roohafza, H. The effects of an educational program based on PRECEDE model on depression levels in patients with coronary artery bypass grafting. ARYA Atheroscler. 2012, 8, 36-42.

51. Horne, D.; Kehler, S.; Kaoukis, G.; Hiebert, B.; Garcia, E.; Duhamel, T.A.; Arora, R.C. Depression before and after cardiac surgery: Do all patients respond the same? J. Thorac. Cardiovasc. Surg. 2013, 145, 1400-1406. [CrossRef]

52. Hweidi, I.M.; Gharaibeh, B.A.; Al-Obeisat, S.M.; Al-Smadi, A.M. Prevalence of depression and its associated factors in patients post-coronary artery bypass graft surgery. J. Res. Nurs. 2018, 23, 76-88. [CrossRef]

53. Kendel, F.; Dunkel, A.; Lehmkuhl, E.; Hetzer, R.; Regitz-Zagrosek, V. Does time spent on household activities or housework stress complicate recovery following coronary artery bypass surgery? Women Health 2008, $48,325-338$.

54. Kendel, F.; Gelbrich, G.; Wirtz, M.; Lehmkuhl, E.; Knoll, N.; Hetzer, R.; Regitz-Zagrosek, V. Predictive relationship between depression and physical functioning after coronary surgery. Arch. Intern. Med. 2010, 170, 1717-1721. [CrossRef] [PubMed]

55. Khoueiry, G.; Flory, M.; Abi Rafeh, N.; Zgheib, M.H.; Goldman, M.; Abdallah, T.; Wettimuny, S.; Telesford, B.; Costantino, T.; McGinn, J.T. Depression, disability, and quality of life after off-pump coronary artery bypass grafting: A prospective 9-month follow-up study. Hear. Lung J. Acute Crit. Care 2011, 40, 217-225. [CrossRef] [PubMed]

56. King, K.M.; Colella, T.J.; Faris, P.; Thompson, D.R. Using the cardiac depression scale in men recovering from coronary artery bypass surgery. J. Clin. Nurs. 2009, 18, 1617-1624. [CrossRef]

57. Korbmacher, B.; Ulbrich, S.; Dalyanoglu, H.; Lichtenberg, A.; Schipke, J.D.; Franz, M.; Schäfer, R. Perioperative and long-term development of anxiety and depression in CABG patients. Thorac. Cardiovasc. Surg. 2013, 61, 676-681. [CrossRef]

58. Kozora, E.; Kongs, S.; Collins, J.; Hattler, B.; Baltz, J.; Hampton, M.; Grover, F.; Novitzky, D.; Shroyer, L. Cognitive outcomes after on- versus off-pump coronary artery bypass surgery. Ann. Thorac. Surg. 2010, 90, 1134-1141. [CrossRef] [PubMed]

59. Macken, L.C.; Yates, B.C.; Meza, J.; Norman, J.; Barnason, S.; Pozehl, B. Health-related quality-of-life outcomes in coronary artery bypass surgery patients and partners. J. Cardiopulm. Rehabil. Prev. 2014, 34, 130-137. [CrossRef]

60. McGrady, A.; McGinnis, R.; Badenhop, D.; Bentle, M.; Rajput, M. Effects of depression and anxiety on adherence to cardiac rehabilitation. J. Cardiopulm. Rehabil. Prev. 2009, 29, 358-364. [CrossRef]

61. McKenzie, L.; Simpson, J.; Stewart, M. The impact of depression on activities of daily living skills in individuals who have undergone coronary artery bypass graft surgery. Psychol. Health Med. 2009, 14, 641-653. [CrossRef]

62. Modica, M.; Castiglioni, P.; Minotti, A.; Faini, A.; Racca, V.; Ferratini, M. Psychological profile in coronary artery by-pass graft patients vs. valve replacement patients entering cardiac rehabilitation after surgery. Sci. Rep. 2018, 8, 14381. [CrossRef]

63. Moser, D.K.; Dracup, K.; Evangelista, L.S.; Zambroski, C.H.; Lennie, T.A.; Chung, M.L.; Doering, L.V.; Westlake, C.; Heo, S. Comparison of prevalence of symptoms of depression, anxiety, and hostility in elderly patients with heart failure, myocardial infarction, and a coronary artery bypass graft. Hear. Lung J. Acute Crit. Care 2010, 39, 378-385. [CrossRef]

64. Murphy, B.; Elliott, P.; Higgins, R.; Le Grande, M.; Worcester, M.; Goble, A.; Tatoulis, J. Anxiety and depression after coronary artery bypass graft surgery: Most get better, some get worse. Eur. J. Cardiovasc. Prev. Rehabil. 2008, 15, 434-440. [CrossRef] [PubMed]

65. Nair, V.V.; Nair, J.T.K.; Das, S.; Singh, K.K.; Kathayanat, J.T.; Radhakrishnan, R.; Chooriyil, N.; Babu, A. Lifestyle practices, health problems, and quality of life after coronary artery bypass grafting. Indian J. Thorac. Cardiovasc. Surg. 2018, 34, 476-482. [CrossRef]

66. Nemati, M.H.; Astaneh, B. The impact of coronary artery bypass graft surgery on depression and anxiety. J. Cardiovasc. Med. (Hagerstown) 2011, 12, 401-404. [CrossRef] [PubMed]

67. Nunes, J.K.V.R.S.; de Figueiredo Neto, J.A.; de Sousa, R.M.L.; Costa, V.L.X.D.C.; Silva, F.M.A.M.; da Hora, A.F.L.T.; da Silva, E.L.C.; Reis, L.M.C.B. Depression after CABG: A prospective study. Braz. J. Cardiovasc. Surg. 2013, 28, 491-497. 
68. Okamoto, Y.; Motomura, N.; Murashima, S.; Takamoto, S. Anxiety and depression after thoracic aortic surgery or coronary artery bypass. Asian Cardiovasc. Thorac. Ann. 2013, 21, 22-30. [CrossRef]

69. Oldham, M.A.; Hawkins, K.A.; Lin, I.H.; Deng, Y.; Hao, Q.; Scoutt, L.M.; Yuh, D.D.; Lee, H.B. Depression predicts delirium after coronary artery bypass graft surgery independent of cognitive impairment and cerebrovascular disease: An analysis of the neuropsychiatric outcomes after heart surgery study. Am. J. Geriatr. Psychiatry 2019, 27, 476-486. [CrossRef]

70. Perrotti, A.; Mariet, A.S.; Durst, C.; Monaco, F.; Vandel, P.; Monnet, E.; Chocron, S. Relationship between depression and health-related quality of life in patients undergoing coronary artery bypass grafting: A MOTIV-CABG substudy. Qual. Life Res. 2016, 25, 1433-1440. [CrossRef]

71. Perrotti, A.; Ecarnot, F.; Monaco, F.; Dorigo, E.; Monteleone, P.; Besch, G.; Chocron, S. Quality of life 10 years after cardiac surgery in adults: A long-term follow-up study. Health Qual. Life Outcomes 2019, 17, 88. [CrossRef]

72. Phillips-Bute, B.; Mathew, J.P.; Blumenthal, J.A.; Morris, R.W.; Podgoreanu, M.V.; Smith, M.; Stafford-Smith, M.; Grocott, H.P.; Schwinn, D.A.; Newman, M.F.; et al. Relationship of genetic variability and depressive symptoms to adverse events after coronary artery bypass graft surgery. Psychosom. Med. 2008, 70, $953-959$. [CrossRef]

73. Pourafkari, L.; Ghaffari, S.; Tajlil, A.; Shahamfar, J.; Hedayati, S.; Nader, N.D. The impact of cardiac rehabilitation program on anxiety and depression levels after coronary artery bypass graft surgery. Cor Vasa 2016, 58, e384-e390. [CrossRef]

74. Rezaei, O.; Komeilian, R.; Fattah, L.; Mandegar, M.H.; Dolatshahi, B. Quality of life and mental disorders in patients after coronary artery bypass grafting. World Appl. Sci. J. 2013, 26, 34-38. [CrossRef]

75. Sandau, K.E.; Lindquist, R.A.; Treat-Jacobson, D.; Savik, K. Health-related quality of life and subjective neurocognitive function three months after coronary artery bypass graft surgery. Hear. Lung J. Acute Crit. Care 2008, 37, 161-172. [CrossRef] [PubMed]

76. Schwarz, N.; Schoenburg, M.; Möllmann, H.; Kastaun, S.; Kaps, M.; Bachmann, G.; Sammer, G.; Hamm, C.; Walther, T.; Gerriets, T. Cognitive decline and ischemic microlesions after coronary catheterization. A comparison to coronary artery bypass grafting. Am. Heart J. 2011, 162, 756-763. [CrossRef] [PubMed]

77. Sorensen, E.A.; Wang, F. Social support, depression, functional status, and gender differences in older adults undergoing first-time coronary artery bypass graft surgery. Hear. Lung J. Acute Crit. Care 2009, 38, $306-317$. [CrossRef] [PubMed]

78. Spezzaferri, R.; Modica, M.; Racca, V.; Ripamonti, V.; Tavanelli, M.; Brambilla, G.; Ferratini, M. Psychological disorders after coronary artery by-pass surgery: A one-year prospective study. Monaldi Arch. Chest Dis. Arch. Monaldi Mal. Torace 2009, 72, 200-205. [CrossRef] [PubMed]

79. Stenman, M.; Sartipy, U. Depression screening in cardiac surgery patients. Hear. Lung Circ. 2019, 28, 953-958. [CrossRef]

80. Thomas, M.; Hariharan, M.; Rana, S. Psychological distress as predictor of adherence and prognosis among patients undergoing coronary artery bypass grafting. J. Indian Acad. Appl. Psychol. 2016, 42, 161-171.

81. Tsai, M.F.; Tsay, S.F.; Moser, D.; Huang, T.Y.; Tsai, F.C. Examining symptom trajectories that predict worse outcomes in post-CABG patients. Eur. J. Cardiovasc. Nurs. 2019, 18, 204-214. [CrossRef]

82. Tully, P.J.; Baker, R.A.; Turnbull, D.; Winefield, H. The role of depression and anxiety symptoms in hospital readmissions after cardiac surgery. J. Behav. Med. 2008, 31, 281-290. [CrossRef]

83. Tully, P.J.; Winefield, H.R.; Baker, R.A.; Turnbull, D.A.; De Jonge, P. Confirmatory factor analysis of the Beck Depression Inventory-II and the association with cardiac morbidity and mortality after coronary revascularization. J. Health Psychol. 2011, 16, 584-595. [CrossRef]

84. Yang, L.; Wang, J.; Zhang, L.Z.; Hou, J.H.; Yuan, X.; Hu, S.; Zheng, Z. Preoperative high-sensitivity $\mathrm{C}$-reactive protein predicts depression in patients undergoing coronary artery bypass surgery: A single-center prospective observational study. J. Thorac. Cardiovasc. Surg. 2012, 144, 500-505. [CrossRef] [PubMed]

85. Yang, P.; Huang, G.; Tsai, C.; Lou, M. Sleep quality and emotional correlates in taiwanese coronary artery bypass graft patients 1 week and 1 month after hospital discharge: A repeated descriptive correlational study. PLoS ONE 2015, 10, e0136431. [CrossRef] [PubMed]

86. Yüksel, V.; Gorgulu, Y.; Cinar, R.K.; Huseyin, S.; Sonmez, M.B.; Canbaz, S. Impact of experiencing acute coronary syndrome prior to open heart surgery on psychiatric status. Braz. J. Cardiovasc. Surg. 2016, 31, 281-286. [CrossRef] [PubMed] 
87. Zimmerman, L.; Barnason, S.; Young, L.; Tu, C.; Schulz, P.; Abbott, A. Symptom profiles of coronary artery bypass surgery patients at risk for poor functioning outcomes. J. Cardiovasc. Nurs. 2010, 25, $292-300$. [CrossRef]

88. Gong, Y.; Han, T.; Yin, X.; Yang, G.; Zhuang, R.; Chen, Y.; Lu, Z. Prevalence of depressive symptoms and work-related risk factors among nurses in public hospitals in southern China: A cross-sectional study. Sci. Rep. 2015, 4, 1-5. [CrossRef]

89. Tully, P.J.; Bennetts, J.S.; Baker, R.A.; McGavigan, A.D.; Turnbull, D.A.; Winefield, H.R. Anxiety, depression, and stress as risk factors for atrial fibrillation after cardiac surgery. Hear. Lung J. Acute Crit. Care 2011, 40, 4-11. [CrossRef]

90. Botzet, K.; Dalyanoglu, H.; Schäfer, R.; Lichtenberg, A.; Schipke, J.D.; Korbmacher, B. Anxiety and depression in patients undergoing mitral valve surgery: A prospective clinical study. Thorac. Cardiovasc. Surg. 2018, 66, 530-536. [CrossRef]

91. Boyer, B.A.; Matour, S.J.; Crittenden, K.B.; Larson, K.A.; Cox, J.M.; Link, D.D. Appraisals of fear, helplessness, and perceived life-threat during emergent cardiac surgery: Relationship to pre-surgical depression, trauma history, and posttraumatic stress. J. Clin. Psychol. Med. Settings 2013, 20, 173-185. [CrossRef]

92. Poole, L.; Ronaldson, A.; Kidd, T.; Leigh, E.; Jahangiri, M.; Steptoe, A. Pre-surgical depression and anxiety and recovery following coronary artery bypass graft surgery. J. Behav. Med. 2017, 40, 249-258. [CrossRef]

93. Murphy, B.; Rogerson, M.; Worcester, M.; Elliott, P.; Higgins, R.; Le Grande, M.; Turner, A.; Goble, A. Predicting mortality 12 years after an acute cardiac event: Comparison between inhospital and 2-month assessment of depressive symptoms in women. J. Cardiopulm. Rehabil. Prev. 2013, 33, 160-167. [CrossRef]

94. Kendel, F.; Dunkel, A.; Müller-Tasch, T.; Steinberg, K.; Lehmkuhl, E.; Hetzer, R.; Regitz-Zagrosek, V. Gender differences in health-related quality of life after coronary bypass surgery: Results from a 1-year follow-up in propensity-matched men and women. Psychosom. Med. 2011, 73, 280-285. [CrossRef] [PubMed]

95. Bjørnnes, A.K.; Parry, M.; Lie, I.; Falk, R.; Leegaard, M.; Rustøen, T. The association between hope, marital status, depression and persistent pain in men and women following cardiac surgery. BMC Womens. Health 2018, 18, 2. [CrossRef] [PubMed]

96. Geulayov, G.; Novikov, I.; Dankner, D.; Dankner, R. Symptoms of depression and anxiety and 11-year all-cause mortality in men and women undergoing coronary artery bypass graft (CABG) surgery. J. Psychosom. Res. 2018, 105, 106-114. [CrossRef] [PubMed]

97. Lie, I.; Arnesen, H.; Sandvik, L.; Hamilton, G.; Bunch, E.H. Predictors for physical and mental health $6 \mathrm{months}$ after coronary artery bypass grafting. A cohort study. Eur. J. Cardiovasc. Nurs. 2010, 9, 238-243. [CrossRef]

98. Patron, E.; Messerotti Benvenuti, S.; Palomba, D. Preoperative and perioperative predictors of reactive and persistent depression after cardiac surgery: A three-month follow-up study. Psychosomatics 2014, 55, 261-271. [CrossRef]

99. Tindle, H.; Belnap, B.H.; Houck, P.R.; Mazumdar, S.; Scheier, M.F.; Matthews, K.A.; He, F.; Rollman, B.L. Optimism, response to treatment of depression, and rehospitalization after coronary artery bypass graft surgery. Psychosom. Med. 2012, 74, 200-207. [CrossRef]

100. Bruce, K.M.; Yelland, G.W.; Smith, J.A.; Robinson, S.R. Recovery of cognitive function after coronary artery bypass graft operations. Ann. Thorac. Surg. 2013, 95, 1306-1313. [CrossRef]

101. Roohafza, H.; Sadeghi, M.; Khani, A.; Andalib, E.; Alikhasi, H.; Rafiei, M. Psychological state in patients undergoing coronary artery bypass grafting surgery or percutaneous coronary intervention and their spouses. Int. J. Nurs. Pract. 2015, 21, 214-220. [CrossRef]

102. Oldham, M.A.; Vachon, J.; Yuh, D.; Lee, H.B. Cognitive outcomes after heart valve surgery: A systematic review and meta-analysis. J. Am. Geriatr. Soc. 2018, 66, 2327-2334. [CrossRef]

103. Haschke, A.; Hutter, N.; Baumeister, H. Indirect costs in patients with coronary artery disease and mental disorders: A systematic review and meta-analysis. Int. J. Occup. Med. Environ. Health 2012, 25, 319-329. [CrossRef]

104. Morone, N.E.; Weiner, D.K.; Belnap, B.H.; Karp, J.F.; Mazumdar, S.; Houck, P.R.; He, F.; Rollman, B.L. The impact of pain and depression on recovery after coronary artery bypass grafting. Psychosom. Med. 2010, 72, 620-625. [CrossRef] [PubMed]

105. Khoury, H.; Sanaiha, Y.; Rudasill, S.E.; Mardock, A.L.; Sareh, S.; Benharash, P. Readmissions following isolated coronary artery bypass graft surgery in the United States (from the Nationwide Readmissions Database 2010 to 2014). Am. J. Cardiol. 2019, 124, 205-210. [CrossRef] [PubMed] 
106. Koivula, M.; Halme, N.; Åstedt-Kurki, P. Predictors of depressive symptoms among coronary heart disease patients: A cross-sectional study nine years after coronary artery bypass grafting. Hear. Lung J. Acute Crit. Care 2010, 39, 421-431. [CrossRef]

107. Sharif, F.; Shoul, A.; Janati, M.; Kojuri, J.; Zare, N. The effect of cardiac rehabilitation on anxiety and depression in patients undergoing cardiac bypass graft surgery in Iran. BMC Cardiovasc. Disord. 2012, 12, 40. [CrossRef]

108. Kabboul, N.; Tomlinson, G.; Francis, T.; Grace, S.; Chaves, G.; Rac, V.; Daou-Kabboul, T.; Bielecki, J.; Alter, D.; Krahn, M. Comparative effectiveness of the core components of cardiac rehabilitation on mortality and morbidity: A systematic review and network meta-analysis. J. Clin. Med. 2018, 7, 514. [CrossRef] [PubMed]

109. Dao, T.K.; Youssef, N.A.; Armsworth, M.; Wear, E.; Papathopoulos, K.N.; Gopaldas, R. Randomized controlled trial of brief cognitive behavioral intervention for depression and anxiety symptoms preoperatively in patients undergoing coronary artery bypass graft surgery. J. Thorac. Cardiovasc. Surg. 2011, 142, e109-e115. [CrossRef] [PubMed]

110. Hare, D.L.; Davis, C.R. Cardiac depression scale: Validation of a new depression scale for cardiac patients. J. Psychosom. Res. 1996, 40, 379-386. [CrossRef]

111. Radloff, L.S. The CES-D Scale a self-report depression scale for research in the general population. Appl. Psychol. Meas. 1977, 1, 385-401. [CrossRef]

112. Nieveen, J.L.; Zimmerman, L.M.; Barnason, S.A.; Yates, B.C. Development and content validity testing of the Cardiac Symptom Survey in patients after coronary artery bypass grafting. Hear. Lung J. Acute Crit. Care 2008, 37, 17-27. [CrossRef]

113. Brown, T.A.; Chorpita, B.F.; Korotitsch, W.; Barlow, D.H. Psychometric properties of the Depression Anxiety Stress Scales (DASS) in clinical samples. Behav. Res. Ther. 1997, 35, 79-89. [CrossRef]

114. Sanavio, S.; Bertolotti, G.; Michielin, P.; Vidotto, G.; Zotti, A. Cognitive Behavioural Assessment 2.0, Scale Primarie; Organizzazioni Speciali: Firenze, Italy, 1987.

115. Sheikh, J.I.; Yesavage, J.A. Geriatric Depression Scale (GDS): Recent evidence and development of a shorter version. Clin. Gerontol. J. Aging Ment. Heal. 1986, 5, 165-173.

116. Hamilton, M. A rating scale for depression. J. Neurol. Neurosurg. Psychiatry 1960, 23, 56-62. [CrossRef] [PubMed]

117. Zuckerman, M.; Lubin, B. Manual for the Multiple Affect Adjective Checklist; Educational and Industrial Testing Service: San Diego, CA, USA, 1965.

118. Kroenke, K.; Spitzer, R.L.; Williams, J.B.W. The patient health questionnaire-2: Validity of a two-item depression screener. Med. Care 2003, 41, 1284-1292. [CrossRef] [PubMed]

119. Kroenke, K.; Spitzer, R.L.; Williams, J.B.W. The PHQ-9: Validity of a brief depression severity measure. J. Gen. Intern. Med. 2001, 16, 606-613. [CrossRef] [PubMed]

120. McNair, D.; Lor, M.; Droppeman, L. Profile of Mood States Manual; Educational and Industrial Testing Service: San Diego, CA, USA, 1992.

121. Zung, W.W.K. A Self-Rating depression scale. Arch. Gen. Psychiatry 1965, 12, 63-70. [CrossRef] [PubMed]

122. Derogatis, L.R. SCL-90-R, Administration, Scoring \& Procedures Manual-II for the Revised Version and Other Instruments of the Psychopathology Rating Scale Series; Clinical Psychometric Research: Townson, MD, USA, 1992.

(C) 2020 by the authors. Licensee MDPI, Basel, Switzerland. This article is an open access article distributed under the terms and conditions of the Creative Commons Attribution (CC BY) license (http://creativecommons.org/licenses/by/4.0/). 